\title{
Crypt-OR: A privacy-preserving system for exemplar-based object-removal over the cloud
}

\author{
Vishesh Kumar Tanwar ${ }^{\mathrm{a}, \mathrm{c}, *}$, Balasubramanian Raman ${ }^{\mathrm{b}, \mathrm{c}}$, Rama Bhargava $^{\mathrm{a}, \mathrm{c}}$ \\ ${ }^{a}$ Department of Mathematics \\ ${ }^{b}$ Machine Vision Lab, Department of Computer Science 85 Engineering \\ ${ }^{c}$ Indian Institute of Technology Roorkee, INDIA
}

\begin{abstract}
Object removal is a technique for removing the undesired object(s) and then fill-in the empty region(s) in an image such that the modified image is visually plausible. The existing algorithms are unable to provide promising results when the region to be removed - has varying textured-neighborhood, is small in size and the depth of the image and, is of specific geometric shapes such as triangle and rectangle. In this paper, we proposed a new algorithm by incorporating the merits of partial differential equations (PDEs) and exemplar-based schemes to address these challenges. The data term, which measures the continuity of isophotes in exemplar-based methods, is modified by incorporating a regularizer term and partial derivatives up to second order of the input image. This regularizer enhances the strength of isophotes striking the boundary and boosts the information propagation in an unbiased manner, in terms of pixel intensity values. Additionally, the low-cost, agility, and accessing flexibility benefits of cloud services have attracted user's attention today. Besides, users are concerned about utilizing them for their data, as they are supported by untrusted third parties. Addressing these privacy concerns for object-removal in an image over the cloud server, we extended and modified our algorithm to make it compatible for $(T, N)$-threshold Shamir secret sharing scheme (SSS). This

\footnotetext{
* Corresponding author

Email addresses: vtanwar@ma.iitr.ac.in (Vishesh Kumar Tanwar), bala@cs.iitr.ac.in

(Balasubramanian Raman), rama.bhargava@ma.iitr.ac.in (Rama Bhargava)
}

Preprint submitted to Journal of ${ }^{A} T_{E} X$ Templates

June 15, 2020 
privacy-preserving system is an end-to-end system for object-removal in the ED over the cloud server namely Crypt-OR. Crypt-OR is evaluated by removing synthetically imposed objects in real-images. Further, Crypt-OR has proved to be secure under various pixel-based cryptographic attacks such as frequency-known attack and pixel-correlation attack.

Keywords: Cloud computing, Image inpainting, Visual cryptography, Encrypted domain, Shamir secret sharing scheme

\section{Introduction}

Over the last decade, numerous images/videos algorithms for object recognition, classification, pose estimation and, semantic segmentation have been proposed Guo et al. (2016); Voulodimos et al. (2018). Besides their high perfor-

5 mances, these schemes are time-consuming and require high-configuration computing resources such as GPUs. To facilitate users, the Cloud Service Providers, hereafter abbreviated as CSP, are providing services such as Platform-as-aservice (PaaS), Infrastructure-as-a-Service (IaaS), and Software-as-a-service (SaaS) for accomplishing their tasks. Azure Machine Learning1 Google Prediction 10 API 2 Amazon Rekognition 3 are few instances. In SaaS, users have to share their private data, which may contain confidential and sensitive information, with CSP to utilize the services. Besides the excellent benefits given by CSPs, users are always concerned about data breaching. The consternation of data storage to CSP has been resolved with Advanced Encryption Standards (AES), 15 as no data, in particular images, can be revealed from their encrypted version Kaur \& Kumar (2018), even with the infinite computational resources. In contrast, processing an encrypted image to achieve the same results as that of plain (non-encrypted) image constitutes a challenging problem, because encryption schemes distort pixels inter-correlation and trichotomy law in pixel-intensities.

\footnotetext{
1 https://azure.microsoft.com/en-in/services/machine-learning/

2 https://cloud.google.com/ai-platform ${ }^{3}$ https://aws.amazon.com/rekognition/
} 


\subsection{Privacy-preserving image processing}

Recently, researchers have presented several image/video processing models to perform different tasks in the ED. Lathey et al. Lathey et al. (2013); Lathey \& Atrey (2015) have proposed a technique for privacy-preserving image enhancement such as noise removal, smoothing, and sharpening, in the ED. The

25 image is secured through $(N, T)$-threshold Secret Sharing Scheme (SSS) Shamir (1979). Shamir's SSS is a homomorphic encryption scheme that partitions a secret $s$ into $N$ shares and requires at-least $T$ shares to reconstruct $s$ losslessly. Individually, each share appears random and contains zero information making the scheme secure. On reconstructing with less than $T$-shares, the resultant „value is equivalent to zero information. Similarly, Mohanty et al. Mohanty et al. (2013) and Tanwar et al. Tanwar et al. (2018a) have presented schemes for privacy-preserving image resizing and cropping in ED over the cloud. The ramp SSS is utilized to secure the user's image from an adversary and interpolation technique over known intensity values to compute unknown pixel-intensities in ED. A scheme for extracting and locating face regions in ED is proposed by Yan and Kankanhali Yan \& Kankanhalli (2015). They secured the image information using coefficients of discrete cosine transform. Rahulamathavan et al. Rahulamathavan et al. (2013) presented a facial expression recognition system in an encrypted image, through Paillier cryptosystem, using local fisher discrim-

40 inant analysis. Sayed et al. SaghaianNejadEsfahani et al. (2012) proposed an information-theoretical wavelet denoising model based on interactive protocols of SSS. They utilized the additive and scalar-multiplicative homomorphisms of Shamir's SSS for basic filtering and thresholding operations that occur during the denoising process. Alberto et al. Pedrouzo-Ulloa et al. (2016) presented a secure image denoising model using ring learning with errors, a lattice-based cryptosystem, which is fully non-interactive in comparison to SaghaianNejadEsfahani et al. (2012).

Hsu et al. Hsu et al. (2012) proposed a model for extracting SIFT features in ED using the Paillier cryptosystem. Chu et al. Chu et al. (2013) presented 50 a real-time object detection model for video surveillance. The scheme trans- 
formed each frame into multiple blocks, and then each block is multiplied by a random matrix. Further, the Gaussian mixture model is used for detecting a moving object(s). The encryption is performed by matrix permutation and color flipping. However, using the same encryption key for the whole process 55 in video surveillance increases the rate of collision attacks. Additionally, Chu et al.'s model is based on each pixel-level operation, which highly increases the computational complexity. In Orlandi et al. (2007), an interactive protocol between the model and the data owners for evaluating the activation functions in the neural network is presented using Homomorphic Encryption (HE). Xie et al. Xie et al. (2014) and Gilad et al. Gilad-Bachrach et al. (2016) proposed privacy-preserving image classification using Fully Homomorphic Encryption (FHE) Rivest et al. (1978) scheme. The utilization of FHE algorithms exponentially increases the computation overhead, making them impractical for real applications.

\subsection{Image inpainting}

It is a process of modifying images such that an unseen observer is unable to differentiate the original and inpainted image. In the ancient period, it was practiced by art-workers for restoring their images to make them "up to date". The objective of image inpainting is to fill the deteriorated region in an image to make them more visually appealing.

Diffusion-based inpainting techniques utilize the propagating-structure of heat flow through diffusion Partial Differential Equations (PDEs), to propagate the local information to the region to be inpainted $\Omega$. An iterative mathematical formulating the technique performed by the professional inpaint art-workers was presented by Bertalmio et al. Bertalmio et al. (2000). The formulation leverage the isophotes-prolongation technique of Masnou and Morel Masnou \& Morel (1998) arriving at the boundary of $\Omega$. The isophotes are propagated in the inward direction in such that they do not cross themselves and generate undesired results. The authors tested their scheme to remove the scratches and overlaid-texts in an image. Following, numerous amount of inpainting models 
incorporating the variants of linear and non-linear isotropic/anisotropic diffusion equations Bertalmio et al. (2001); Peter et al. (2016) have been proposed. These schemes are capable of completing the curves and small-sized objects; however, they generate smoothing/blurring effects for large and textured regions.

These perplexities have been addressed in Criminisi et al. (2004); Wang et al. (2013); Xiang et al. (2019) using exemplar-based texture synthesis approach presented by Efros and Leung Efros \& Leung (1999). These schemes scan the source region $\Phi$ to obtain the best-exemplar patches for the region $\Omega$, determined by similarity metric. Then, these patches are copied in the respective positions in $\Omega$. Because of the "No Free Lunch Theorem", these schemes are found to output unconnected edges. To connect these edges, researchers have combined theories of the categories mentioned above and proposed hierarchical schemes which improve perceptual and visual plausible Drori et al. (2003); Arias et al. (2011). Recently, the data-driven and parameter learning-based schemes using 95 Convolutional Neural Networks (CNNs) have been proposed Nazeri et al. (2019); Yu et al. (2018). Nazeri et al. Nazeri et al. (2019) scheme is a two-stage approach, i.e., structure prediction and image completion. Both of these steps utilize the adversarial model, a variant of deep learning models. The scheme hallucinates the edges of the regions to be inpaint and then utilizes these edges to fill-in the region for image completion. Yu et al. Yu et al. (2018) scheme is a generative model, based upon feed-forward convolutional neural networks (CNNs), for synthesizing image structure and surrounding image features. Their model is evaluated to fill-in multiple only square-shaped holes irrespective of size and location. However, these learning models require a huge amount of training data and computational resources for significant results. For a detailed overview of image inpainting, ref. Guillemot \& Le Meur (2013).

Nowadays, CSPs are providing inpainting SaaS such as Webpaint ${ }^{1}$ and Photoscissor: $2^{2}$, They require the user's image in a plausible visual form, which increases the concerns towards the protection of image information. Moreover,

1 https://www.webinpaint.com/ 2 https://online.photoscissors.com/ 
the existing inpainting techniques cannot be directly implemented in ED due to their dependency on neighboring pixels, and distortion occurred through encryption techniques.

In the context of image inpainting, Yan et al. Yan et al. (2018) proposed a partial secret image sharing scheme for hiding the desired region of the image rather than the whole image. Their technique fills the target region by using the inpainting technique in $\mathrm{PD}$, which reveals the information of non-inpainting or source region while inpainting is performed over the cloud. We address above concerns and propose Crypt-OR, a privacy-preserving end-to-end system for removing object(s), specified by the user, in ED.

\subsection{Contributions}

The key contributions of the paper are as follows -

1. A new image inpainting scheme for object-removal is proposed. The data term, which measures the continuity of isophotes in exemplar-based methods, is introduced by integrating a regularizer and partial derivatives up to second order of the input image. These derivatives improve the propagation efficiency of texture and structural information from the source region to the target region in the isophotes directions. The priority function is defined as the linear function of confidence and data terms.

2. The proposed scheme is extended to develop a privacy-preserving objectremoval model, Crypt-OR, for removing pre-defined undesired object(s) and fill-up the scratches occurred in images in the ED over the cloud infrastructures. The model encourages CSP to search the best-exemplar corresponding to the query patch in ED. The governing equations of inpainting in $\mathrm{PD}$ are transformed to make them compatible with the homomorphic properties of Shamir's SSS.

3. The scheme has proved to be secure under various cryptographic attacks such as Frequency-known attack and Pixels-correlation attack. Crypt-OR does not depend upon the topological shape of the region to be inpainted. 


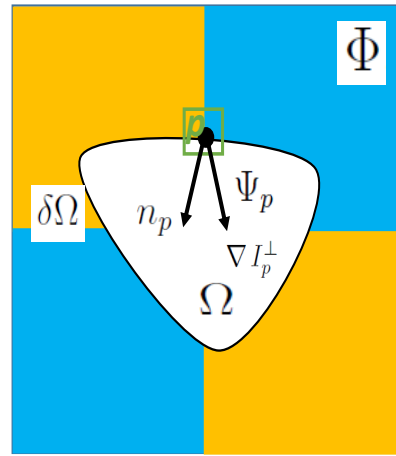

(a)

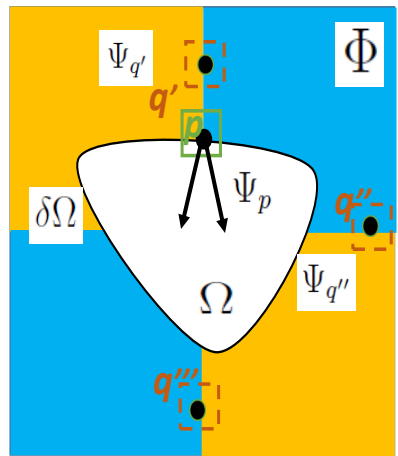

(b)

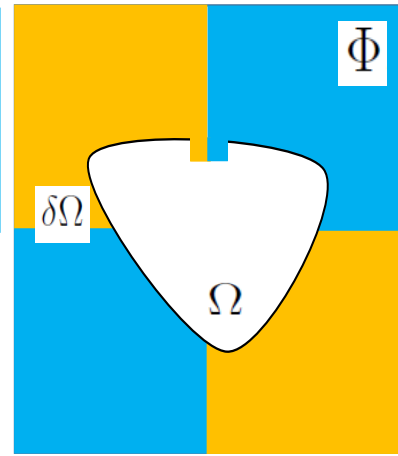

(c)

Figure 1: A general framework of exemplar-based image inpainting. (a) Original image $I$ with region to be inpaint $\Omega$ and source region $\Phi$. (b) It represents the possible patch candidates (in red) corresponding to the patch patch with center $p$ (in green), (c) inpainted image.

The proposed model is evaluated over various images with the different region(s). The PSNR, SSIM, ASVS, and DN measures are reported, which are found to be very close to that of the existing scheme in PD.

4. To best of our knowledge, Crypt-OR is the first move towards removing objects in a given image in the ED using a search-copy-paste approach incorporating the cloud infrastructure(s).

Organization: Section 3 is devoted to describing Shamir's SSS and the fundamentals of the exemplar-based scheme. The proposed framework for Crypt-OR is defined in Section 4 and, security analysis in Section 5 . The qualitative and quantitative analysis of the proposed scheme are provided in Section 6, followed by discussion and analysis in Section 7 . Finally, Section 8 concludes the paper.

\section{Related Work}

Over the recent decades, researchers have proposed various privacy preserving schemes for processing multimedia Bost et al. (2015); Lathey et al. (2013); 
Jha et al. (2005); Orlandi et al. (2007). We discuss a glimpse of privacy preserving models for various image processing and other Machine Learning (ML) tasks. A detailed survey for preserving visual data was presented by Padilla-López et al. Padilla-López et al. (2015).

\subsection{Privacy-preserving processing}

The existing privacy preserving systems address the tasks of enhancement and feature extraction Lathey et al. (2013); Lathey \& Atrey (2015); Tanwar et al. (2018b) over the ED. The scheme proposed by Lathey et al. Lathey et al. (2013); Lathey \& Atrey (2015) is efficient for noise removal, edge sharpening, unsharp masking and anti-aliasing from an image in ED. The image is secured through „N, $N)$-threshold Shamir Secret Sharing (SSS) scheme. Tanwar et al. Tanwar et al. (2018b) proposed a privacy preserving algorithm for image scaling over the cloud in which image is encrypted by ramp SSS. They utilized 2D-Bicubic interpolation to interpolate the new pixel intensity values over cloud shares. A novel scheme for extracting and locating face regions in ED is proposed by Yan and Kankanhali Yan \& Kankanhalli (2015). The information of image is preserved using coefficients of discrete cosine transform. Rahulamathavan et al.

170 Rahulamathavan et al. (2013) presented a facial expressions recognition system in ED using local fisher discriminant analysis. The input image is encrypted using Paillier cryptosystem.

\subsection{Privacy-preserving clustering}

Clustering is an unsupervised ML task, which aims to partition a group of $D$-dimensional data points into $K$ different subgroups, formally known as clusters, based on similarity metric. Researchers have proposed privacy preserving models for performing clustering in ED for vertically Jha et al. (2005); ? and arbitrary ?? partitioned data. Zhang et al. ? proposed a possibilistic c-means algorithm for fuzzy clustering of FHE encrypted big data. Xing et al. Xing et al. (2017) proposed a two-stage protocol for privacy preserving $k$-means clustering based on Paillier cryptosystem over social networking big-data. 


\subsection{Privacy-preserving recognition}

As discussed earlier, various models for accomplishing recognition tasks while preserving data privacy have been presented Ma et al. (2018); Yonetani et al.

185 Bost et al. (2015); Shokri \& Shmatikov (2015).

Authors in Xie et al. (2014); Gilad-Bachrach et al. (2016) proposed FHEbased Rivest et al. (1978) classification schemes which provides results similar to plain domain. The utilization of FHE techniques exponentially increase the computation overhead making these schemes impractical. In Orlandi et al. (2007), an interactive protocol between the model and data owners for evaluating the activation functions in neural network is presented using Homomorphic Encryption (HE). Bost et al. Bost et al. (2015) proposed a scheme for Naive Bayes, Decision Trees and Hyperplane Decision classifiers. The authors combined FHE encrypting data. In comparison with existing schemes Xie et al. (2014); GiladBachrach et al. (2016); Bost et al. (2015), SecureDL is more computation and communication efficient.

\section{Preliminaries}

\subsection{Shamir $S S S$}

The scheme proposed by Adi Shamir Shamir (1979) states that if a secret value $s$, is shared into $N$ meaningless shares, then $T$ or more shares are required for lossless reconstruction of $s$, where $T$ and $N$ are positive integers such that $T \leq N$. The shares of $s$ are generated via a polynomial $g(x)$ of degree $(T-1)$, defined as:

$$
g(x)=\left(s+c_{1} x+c_{2} x^{2}+\ldots+c_{T-1} x^{(T-1)}\right) \bmod \Upsilon
$$

where the coefficients $c_{1}, \ldots, c_{T-1}$ are randomly chosen from a Galois field of prime order $\Upsilon$, denoted as $\mathbb{G} F(\Upsilon)$ and $x \epsilon[1, N]$ is an integer. 
The reconstruction of $s$ from its $N$ shares say $\left\{S_{1}, S_{2}, \ldots, S_{N}\right\}$ is accomplished using Lagrange's interpolation over more than $T-1$ shares. Moreover, the Shamir's SSS has been proved to be a correct and perfect, in the sense that - (a) out of $N$ shares, $T$ or more shares can reconstruct the secret value losslessly, (b) the zero information will reveal if reconstruction is performed using less than $T$ shares. Also, Shamir's SSS is homomorphic with respect to addition and scalar multiplication.

\subsection{Exemplar-based technique - Fundamentals}

Consider an image $I$, in which user aims to remove and fill-in the target region $\Omega$, using the source region $\Phi$, where $\Phi=I-\Omega$. The boundary between these regions is denoted by $\partial \Omega$, as depicted in Fig. 11(a). For pixel $p, \Psi_{p}$ indicates a patch or window of size $w \times w$ centered at $p$, as presented in Fig.

1(a). The standard rule-of-thumb in exemplar-based inpainting consists three steps as follows:

\subsubsection{Computing patch priorities}

For pixel $p \epsilon \partial \Omega$, the priority value $P(p)$ of patch $\Psi_{p}$ is evaluated as a product of integral powers of confidence term $C(p)$ and a data term $D(p)$ as -

$$
\begin{aligned}
P(p) & =[C(p)]^{\alpha}[D(p)]^{\beta}, \quad \alpha, \beta \in \mathbb{N} \\
C(p) & =\frac{\sum_{q \epsilon \Psi_{p} \bigcap(I-\Omega)} C(q)}{\#\left(\Psi_{p}\right)} \text { and } D(p)=\frac{\left|\Delta I_{p}^{\perp} \cdot \overrightarrow{n_{p}}\right|}{\Gamma}
\end{aligned}
$$

where $\#\left(\Psi_{p}\right)$ and $\Gamma$ indicate the number of pixels in $\Psi_{p}$ and normalization factor respectively. The term $C(p)$ is considered as the amount of reliable and meaningful information surrounding the pixel $p$. The $D(p)$ term measures the continuity of isophotes in their direction and helps to improve the priority of patch along the isophotes direction. $D(p)$ is calculated as a vector product of

230 intensity gradient in an orthogonal direction $\Delta I_{p}^{\perp}$ and the normalized vector $\overrightarrow{n_{p}}$, of the binary mask $M$ depicting $\Phi$ and $\Omega$ only, as shown in Fig. 1(a). 


\subsubsection{Finding optimal patch}

Among all patches centered on $\delta \Omega$ i.e., $\left\{\Psi_{p_{1}}, \Psi_{p_{2}}, \ldots, \Psi_{p_{L}}\right\}$, the patch $\Psi_{p}$ having maximum priority value $P(p)$ corresponding to pixel $p$ is considered.

235 Then, the technique searches a patch say $\Psi_{q^{\prime}}$, in $\Phi$ having maximum similarity measure with $\Psi_{p}$ as shown in Fig. 1(b).

\subsubsection{Propagation of source information}

Once the optimal patch $\Psi_{q^{\prime}}$ is obtained, the unknown pixel values in $\Psi_{p}$ are replaced with the corresponding values in $\Psi_{q^{\prime}}$. It can easily be observed in Fig.

240 11(c). Finally, confidence values of unknown pixels are updated through

$$
C(p)=C\left(p^{\prime}\right) \quad \forall \quad p \in \Psi_{q^{\prime}} \bigcap \Omega
$$

\section{Proposed methodology}

The proposed Crypt-OR removes the undesired object(s) and fills in the scratches in an image using an exemplar-based inpainting approach. In contrast with the classical data term and priority function, an Improved priority function, denoted as $P_{i m p}(p)$, and Regularized data term, denoted by $D_{\text {reg }}(p)$ respectively at pixel $p$ are introduced. As discussed earlier, the schemes of PD cannot be directly applied to encrypted images. Therefore, the mathematical equations are modified depending upon the homomorphic operations, addition and scalar multiplication, of Shamir's SSS. Further, a protocol between the user and CSP is provided to accomplish the inpainting task at cloud servers.

In Crypt-OR, two entities are involved: (i) User: who aims to outsource his image $I$ for removing the undesired region, denoted by $\Omega$, in a secure manner and, (ii) CSP: who is "honest-but-curious" and accomplishes the task of inpainting in the encrypted share over the cloud server. CSP maintains the user. The functions to be used are - 
- MaskForm(): The input of this function is an image $I$ in which the region $\Omega$ is highlighted with a specific color say red. The highlighting can easily be performed using Microsoft paint. It outputs a binary mask $M$ indicating $\Omega$ and the source region $\Phi$.

- DeriVal(): This function inputs $I$ and $M$, thereby computing their partial derivatives upto second-order with respect to spatial variables.

- PatchEval(): It takes the priority values $\left\{P\left(p_{z}\right)\right\}_{z=1}^{Z}$ for pixels $\left\{p_{z}\right\}_{z=1}^{Z}$ in $\delta \Omega$ and outputs the most suitable patch.

- UpdateVal(): It updates the regularized data term $D_{\text {reg }}(p)$ and confidence value $C(p)$ for pixel $p$ in $\Omega$.

- ShareGen(): It takes image $I$ and the parameters $(N, T)$ of Shamir's SSS as input and, generates $N$ obfuscated shares say $S_{1}, S_{2}, \ldots, S_{N}$.

\subsection{Regularized data term}

The proposed data term, $D_{\text {reg }}(p)$, is defined as -

$$
D_{\text {reg }}(p)=\left\|D_{1}(p)\right\|+\left\|D_{2}(p)\right\|+\left\|D_{3}(p)\right\|+\lambda \sqrt{\|\mathcal{R}\|}
$$

the term $\sqrt{\|\mathcal{R}\|}$ indicates the introduced regularizer, and

$$
\begin{gathered}
D_{1}(p)=\left[\frac{-\partial I(p)}{\partial y}, \frac{\partial I(p)}{\partial x}\right] \odot\left[\frac{\partial M(p)}{\partial x}, \frac{\partial M(p)}{\partial y}\right] \\
D_{2}(p)=\left[\frac{-\partial^{2} I(p)}{\partial y^{2}}, \frac{\partial^{2} I(p)}{\partial x^{2}}\right] \odot\left[\frac{\partial^{2} M(p)}{\partial x^{2}}, \frac{\partial^{2} M(p)}{\partial y^{2}}\right] \\
D_{3}(p)=\left[\frac{-\partial^{2} I(p)}{\partial x \partial y}, \frac{\partial^{2} I(p)}{\partial y \partial x}\right] \odot\left[\frac{\partial^{2} M(p)}{\partial y \partial x}, \frac{\partial^{2} M(p)}{\partial x \partial y}\right] \odot C \\
\mathcal{R}=\left[\lambda_{1}, \lambda_{2}\right] \odot\left[\left(\frac{\partial M(p)}{\partial x}\right)^{2},\left(\frac{\partial M(p)}{\partial y}\right)^{2}\right]
\end{gathered}
$$


where, $C=[0.5,0.5], \lambda$ is a constant, $\odot$ represents the Hadamard matrix product, $\|$.$\| is a function which sums the entries of the input vector and \frac{\partial L(p)}{\partial t}$ is rate of change of intensity values in $L \epsilon\{I, M\}$ in spatial direction $t \epsilon\{x, y\}$ at

275 pixel $p$. Similarly, $\frac{\partial^{2} L(p)}{\partial x^{2}}$ and $\frac{\partial^{2} L(p)}{\partial y^{2}}$ represent the acceleration of intensity flow in horizontal and vertical direction respectively. The mixed derivative term $\frac{\partial^{2} L(p)}{\partial x \partial y}$ depicts the rate of change of $L$ in $x$-direction followed by $y$-direction. Intuitively, it shows the changes occurred in non-linear curves.

Each term presented in $D_{\text {reg }}$ has different significance in propagating the information in isophotes direction. The term $D_{1}(p)$ computes the maximum orthogonal rate of change of intensity values in each spatial direction of $I$, towards the front-line of boundary $\partial \Omega$. It only incites to incorporate the linear structures for information propagation which may fail to work in real-time images. $D_{2}(p)$ aims to evaluate the magnitude of maximum change in the neighborhood of $p$ in vertical and both horizontal directions of the respective gradients. This term helps to find the direction of edges and sharp changes in $I$. The third term $D_{3}(p)$ motivates to evaluate the curves in the diagonal and cross-diagonal directions. It is important to realize that $D_{\text {reg }}$ contains partial derivatives of second order which boosts data term and hence, improved priority function, $P_{i m p}(p)$, to detect non-linear curves in $I$. Finally, the regularizer term boosts the information propagation in an unbiased manner, in terms of pixel intensities.

\subsection{Improved priority function}

In previous works, priority function for pixel $p$ is given as the product of integral powers of $D(p)$ and $C(p)$ such as in Eq. 2. Experimentally, it is observed that $C(p)$ is inversely proportional to the exponential power of iteration number and hence, approaches to zero for large number of iterations Cheng et al. (2005). This makes the priority value $P(p)$, as defined in Eq. 2, to tend zero without considering the $D(p)$ value and thus, calculation of $P(p)$ becomes meaningless. Due to this, the scheme propagates the undesirable structure and texture information in the target region. Also, each pixel-intensity value is highly inter-correlated with its neighboring pixels and most likely information 


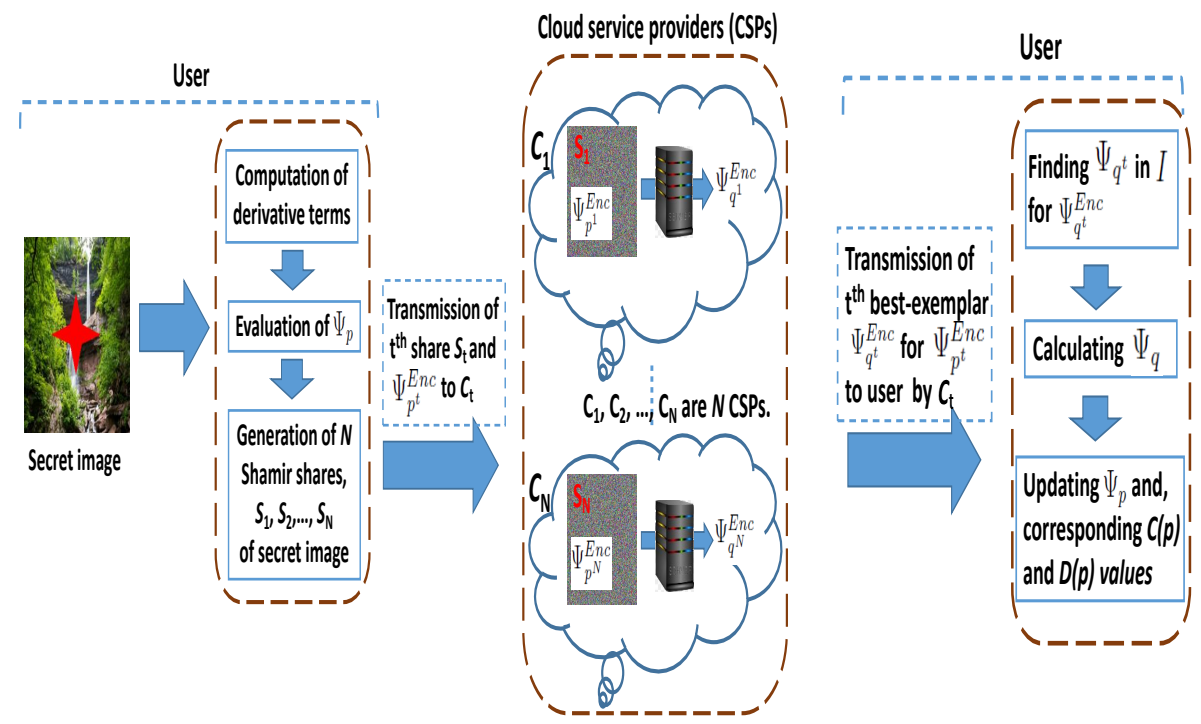

Figure 2: The pictorial representation of Crypt-OR.

lies in the isophotes direction. Additionally, the neighboring information of the target patch for the continuous and meaningful propagation of structure and texture information is considered. In our proposed work, the improved priority function for a pixel $p$, denoted by $P_{i m p}(p)$, is defined as a linear operator of $C(p)$ and $D_{r e g}(p)$ as defined in Eq. 2 and Eq. 4 respectively. The mathematical equation is:

$$
\begin{array}{ll}
P_{\text {imp }}(p) & =\left[\alpha_{1} \times C(p)+\beta_{1}\right]+\left[\alpha_{2} \times D_{\text {reg }}(p)+\beta_{2}\right] \\
\text { or } & =\alpha_{1} \times C(p)+\alpha_{2} \times D_{\text {reg }}(p)+\left(\beta_{1}+\beta_{2}\right) \\
\text { or } & =\alpha_{1} \times C(p)+\alpha_{2} \times D_{\text {reg }}(p)+\beta_{3}
\end{array}
$$

where $\beta_{3}=\left(\beta_{1}+\beta_{2}\right)$ and $\alpha_{i}, \beta_{i}$ are arbitrary hyper-parameters whose values are evaluated through experiments. The value of hyper-parameters are not equal which leads to a non-uniform contribution by each of $C(p)$ and $D_{\text {reg }}(p)$ to $P(p)$. Moreover, $\beta_{3}$ helps to maintain the existence of $P(p)$ with non-zero value when number of iterations increase. 


\subsection{Step-by-step description}

In this section, we present the protocol of Crypt-OR which clearly explains

315

$$
\begin{gathered}
\frac{\partial^{2} I(p)}{\partial x \partial y} \approx \frac{I_{x-2, y}-2 \times I_{x, y}+I_{x+2, y}}{4} \\
\frac{\partial^{2} I(p)}{\partial x^{2}} \approx \frac{I_{x-1, y-1}-I_{x+1, y-1}-I_{x-1, y+1}+I_{x+1, y+1}}{4}
\end{gathered}
$$

where $I_{x, y}$ indicates intensity value of $I$ at position $(x, y)$. Shamir's SSS is not homomorphic in multiplication and division operations, so, Eqs. 10 - 12 will not provide same results as obtained in PD, when performed in ED. The 330 shares are generated using modulo operation with prime $\Upsilon$ as defined in Eq. 1 . Therefore, the above equations are modified as -

$$
\frac{\partial I(p)}{\partial x} \approx \bmod \left[\left(I_{x-1, y}-I_{x+1, y}\right)+\Upsilon, \Upsilon\right]
$$




$$
\frac{\partial^{2} I(p)}{\partial x \partial y} \approx \bmod \left[\left(I_{x-2, y}-2 \times I_{x, y}+I_{x+2, y}\right)+\Upsilon, \Upsilon\right]
$$

Similarly, other derivatives can be approximated with modulo operation. The square root contained in regularizer in Eq. 8 is approximated by Taylor series upto first order. Further, the confidence and data values for each pixel of $I$ are initialized randomly using $\operatorname{ConfVal}()$ and DataVal() respectively.

Step 2: Here, user computes the partial derivatives of mask $M$ upto second order using DeriVal(). It can be observed that these derivative values of $M$ are zero inside the regions $\Omega$ and $\Phi$, albeit, non-zero at the boundary $\partial \Omega$. Moving ahead, the priority values for each $p \epsilon \partial \Omega$, is calculated using Eq. 9, under modulo operation. A pixel $p$ having maximum priority value $P(p)$ is considered to start with and the corresponding patch $\Psi_{p}$ of window size $w \times w$. Then, user operates ShareGen() to generate $N$ shares say $\left\{S_{1}, S_{2}, \ldots, S_{N}\right\}$ of $I$ with prime $\Upsilon$. It is observed that each share $S_{t}$ depends upon random numbers $c_{j}$ 's (Eq. 1), therefore, intensity values in $\Psi_{p}$ changes accordingly. Let $\Psi_{p^{t}}^{E n c}$ denotes the patch $\Psi_{p}$ in share $S_{t}$. Note that $\Psi_{p^{t}}^{E n c}$ varies with $\Psi$ in terms of intensity values only, although, positions will unchanged. Now, the share $S_{t}$ along with the patch $\Psi_{p^{t}}^{E n c}$ will be transmitted to CSP $C_{t}$, for $t=1,2, \ldots, N$.

Step 3: After successfully receiving share $S_{t}$ and $\Psi_{p^{t}}^{E n c}$, CSP $C_{t}$ searches the best possible encrypted patch say $\Psi_{q^{t}}^{E n c}$ similar to $\Psi_{p^{t}}^{E n c}$. The searching is accomplished by defining a difference measure $G()$ between $\Psi_{p^{t}}^{E n c}$ and $\Psi_{q_{l}^{t}}^{E n c}$, for some $l$, in $S_{t}$ as follows -

$$
\begin{gathered}
G_{l}=2 \times \operatorname{Err}_{l}+\Upsilon+(p-1) \\
\text { where, } \operatorname{Err}_{l}=\sum_{k=1}^{w^{2}}\left[\Psi_{q_{l}^{t}}^{E n c}(k)-\Psi_{p^{t}}^{E n c}(k)\right]
\end{gathered}
$$

Note that $\Psi_{q_{l}^{t}}^{E n c}$ is of dimension $w \times w$ contained in $\Phi^{E n c}$, source region in $S_{t}$ corresponding to the source region $\Phi$ of $I$. A patch having least difference value is considered as the best-exemplar $\Psi_{q^{t}}^{E n c}$ corresponding to $\Psi_{p^{t}}^{E n c}$ in $S_{t}$. Then, $C_{t}$ transmits back the obtained patch to user. 
Step 4: User re-collect all $N$ encrypted best-exemplar patches $\left\{\Psi_{q^{l}}^{E n c}\right\}_{l=1}^{N}$ corresponding to $\left\{\Psi_{q^{l}}^{E n c}\right\}_{l=1}^{N}$ obtained from $N$ CSPs. Then, each encrypted patch $\Psi_{q^{t}}^{E n c}$ is decrypted to a patch in PD say $\Psi_{q^{t}}$. It can be clearly concluded that for $t_{1} \neq t_{2}, \Psi_{q^{t_{1}}}$ may be different from $\Psi_{q^{t_{2}}}$. Therefore, the most suitable patch $\Psi_{q}$ with respect to $\Psi_{p}$ is procured by computing structural similarity value between each $\Psi_{q^{t}}$ and $\Psi_{p}$. The patch having maximum similarity value is assumed to be best-exemplar $\Psi_{q}$. The unknown pixels of $\Psi_{p}$ in $\Omega$ are replaced with the corresponding values of $\Psi_{q}$. As defined in Section 3.2 , the data term $D_{\text {reg }}(p)$ and confidence values $C(p)$ are updated with the corresponding $C(p)$ 345 and $D_{\text {reg }}(p)$ values of $\Psi_{p}$.

Step 5: Repeat Step 2 to Step 5 until the intensity values of all the pixels in $\Omega$ are obtained.

\section{Security analysis}

Here, the security analysis of Crypt-OR from an adversary is evaluated through standard image encryption tests Cao et al. (2018).

\subsection{Frequency-known attack}

In this attack, an adversary is given the frequencies of each pixel intensity value, known as frequency histogram, of an encrypted image $\left(I_{E n c}\right)$, but not of the corresponding image $I$ in PD. An adversary manages to extract the frequency histogram of $I$ for given frequency histogram of $I_{E n c}$. The histogram of an image features the contrast, brightness and saturation effects of the contents. The attack demonstrates the change in diffusion and confusion characteristics of $I_{E n c}$ in comparison with $I$. It is assumed that adversary has infinite amount of time and computational resources. In order to secure information in $I$ from the adversary, frequency histogram of $I_{E n c}$ must be significantly different from $I$ and uniform as much as possible.

We consider the Lena image in RGB color-space, $I$, of dimension $256 \times 256$ and its respective red, green and blue channels histograms as depicted in Fig. 

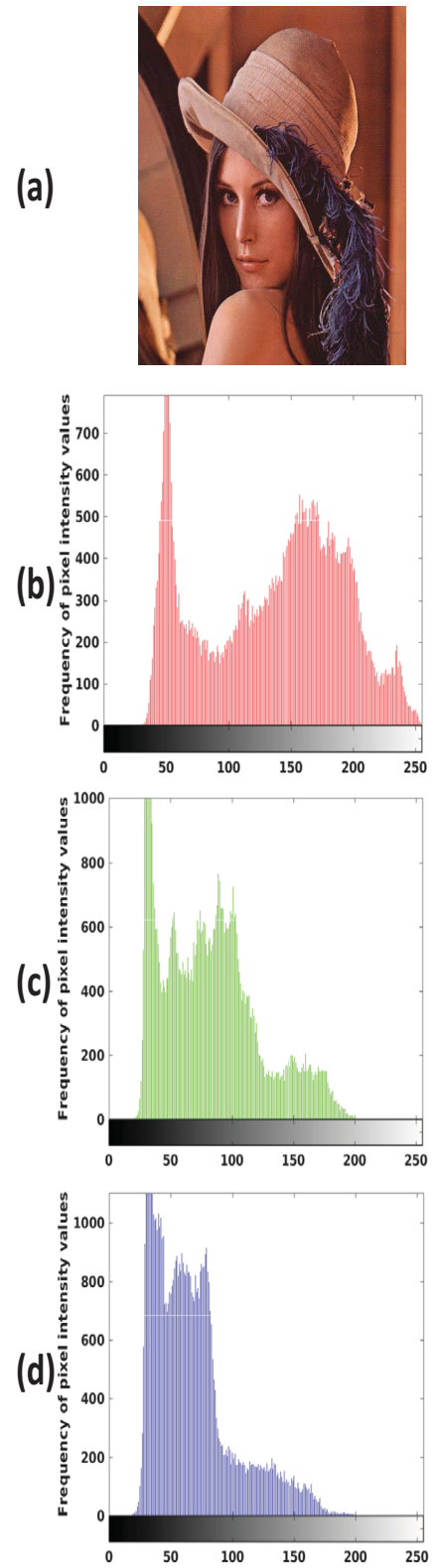
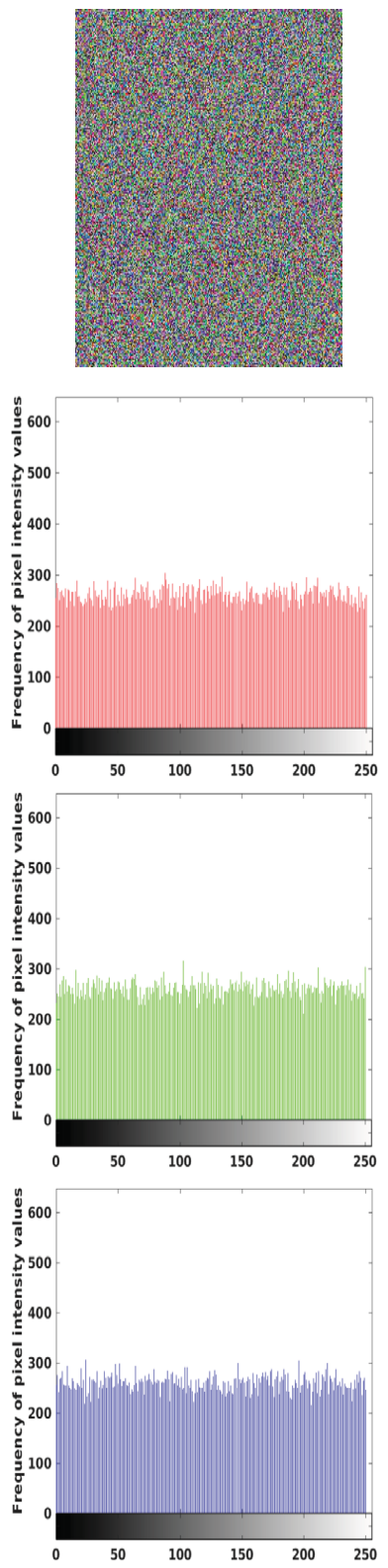
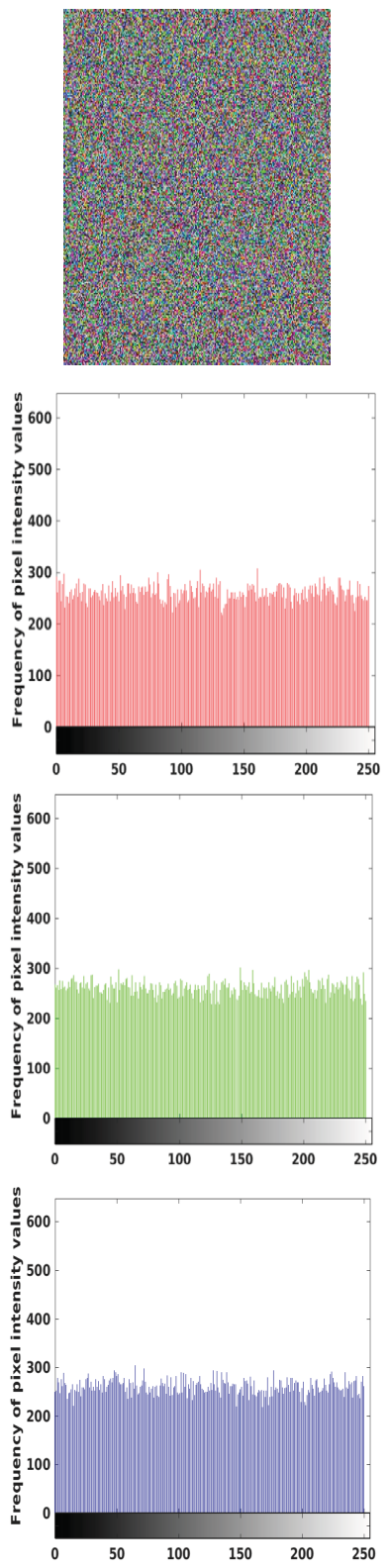

Figure 3: Histogram analysis of Lena RGB-image in PD and its encrypted shares.

3(a). The encrypted shares $I_{S_{1}}$ and $I_{S_{2}}$ of $I$ under modulo $\Upsilon=251$ are depicted 365 in Fig. 3(b) and Fig. 3(c) respectively. It can be observed that histogram of 
(a)
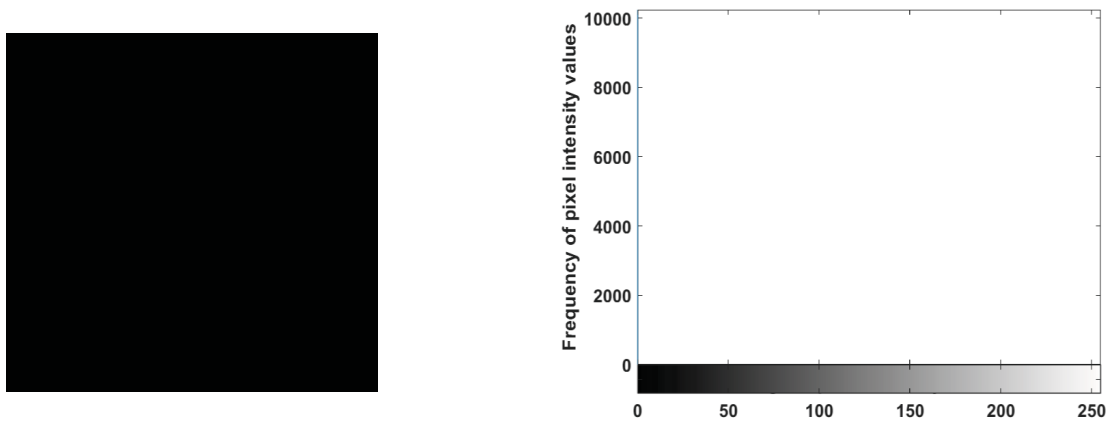

(b)
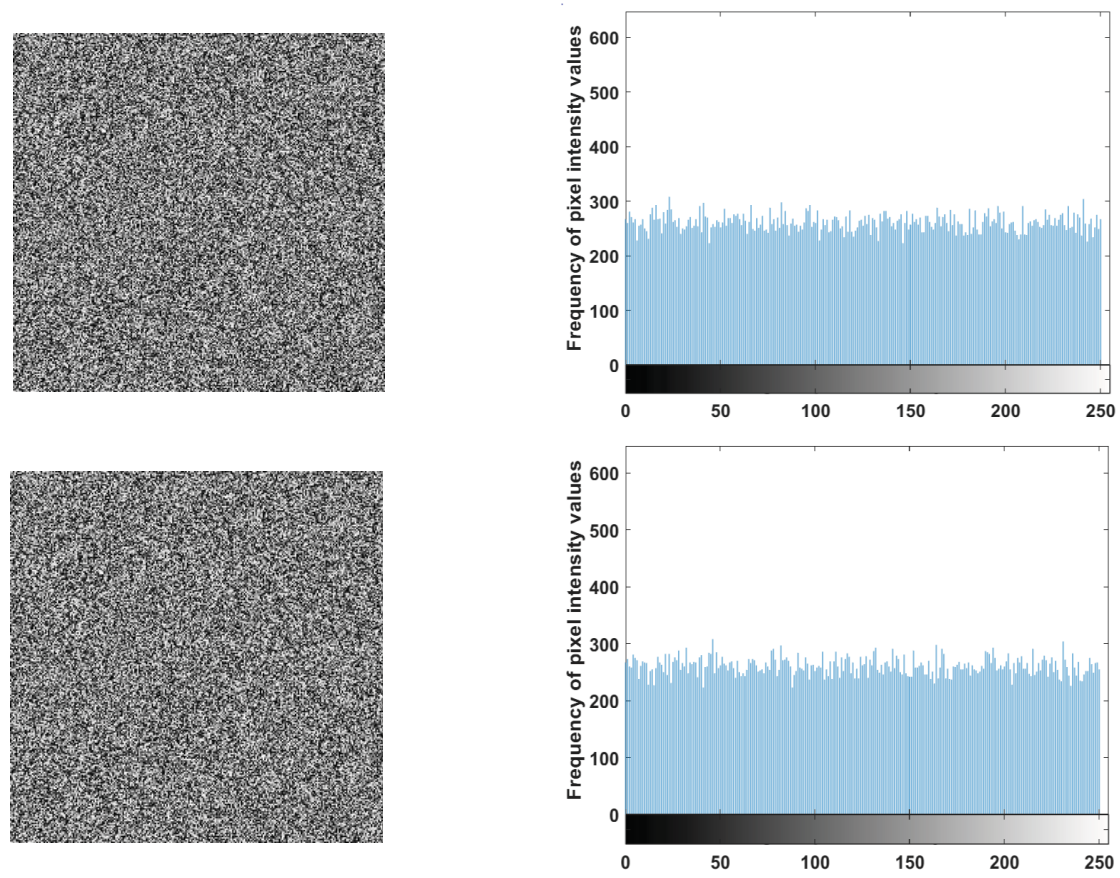

Figure 4: Histogram analysis of z-black image in PD and its encrypted shares.

each channel in PD contains multiple valley-like regions and pixel intensities lie in the range $[30,255]$ (zoom-in horizontal axis). In contrast, the histograms of shares are different than that of secret image and almost uniform. They ranges nearly equal in interval $[0,251]$ (zoom-in horizontal axis), which means theat 370 the pixels information is uniformally distributive over the whole range. Thus, CSPs can extract approximately zero information of user's secret image. 

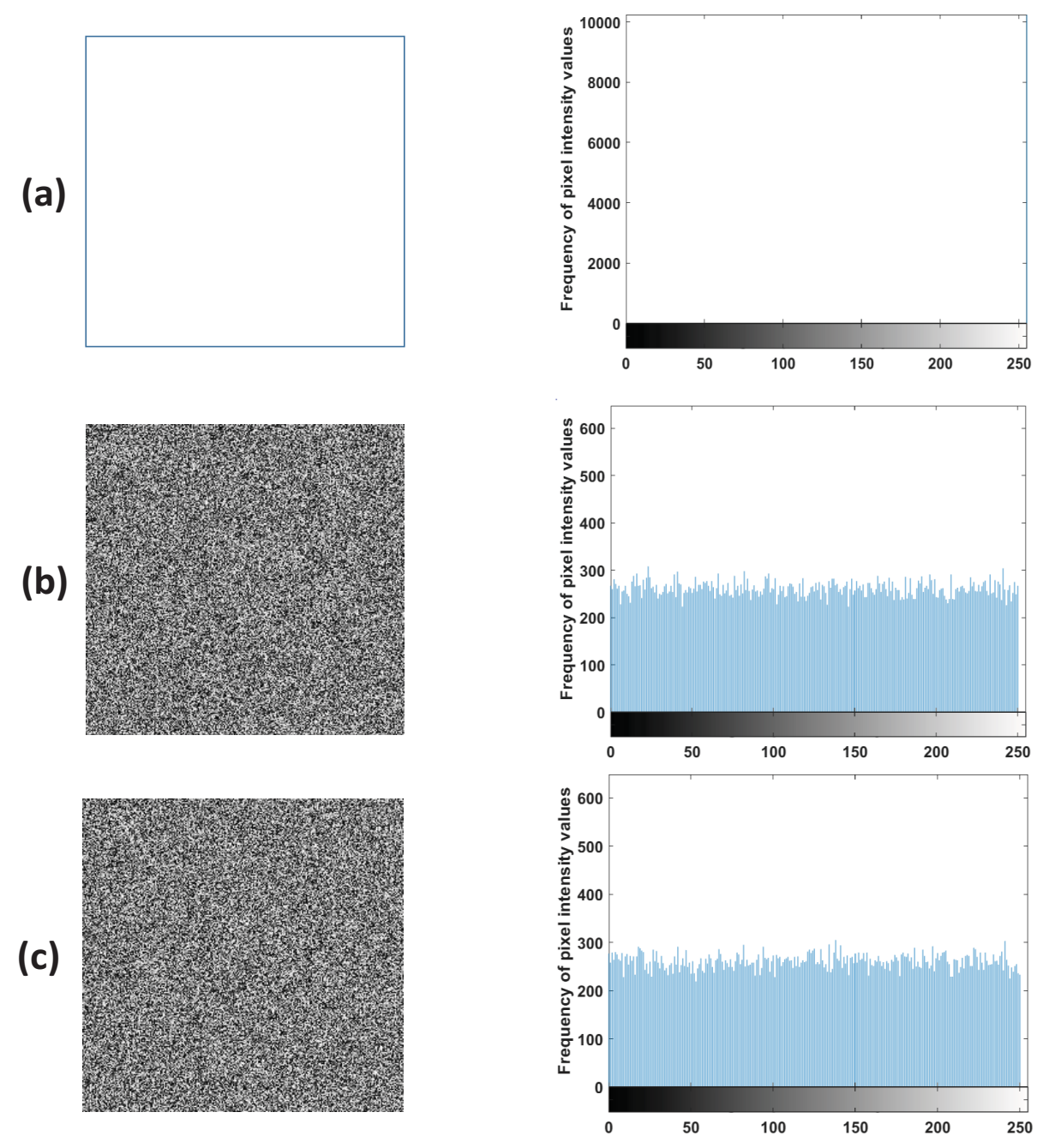

Figure 5: Histogram analysis of white image in PD and its encrypted shares.

Moreover, we perform encyption experiments for an image whose all pixel instensity values are equal i.e., constant image. An encryption scheme is assumed to be completely secure if its output does not depend upon the input values. In

other words, the encrypted form of a constant image and real-time image say Lena are almost same. For this, a z-black and white images are considered as shown in Figs. 4 and 5 . In each Figs. 4 and 5 , (a) contains the origial image and 
the corresponding encrypted shares under modulo $\Upsilon=251$. The corresponding histograms are presented in (b). Note that the histograms of black and white images conatin single spike to 0 and 255 respectively. However, their histograms are distributed uniformly over the range $[0,251]$, proving the complete security of image information.

\subsection{Pixels-correlation attack}

The correlation between neighboring pixels in an image $I$ depict a statistical relevance Chen et al. (2004). High pixels-correlation indicates the high-visual quality of an image, which helps to extract meaningful information. To prevent the information poses by $I$ from an adversary, the correlation value must be low for its encrypted shares, which we analyze for Crypt-OR.

\subsubsection{Numerical values}

The correlation of $K$ neighboring pixels $\left\{\left(x_{1}, y_{1}\right),\left(x_{2}, y_{2}\right), \ldots,\left(x_{K}, y_{K}\right)\right\}$ for a gray-scale image $I$, denoted by corr, is evaluated as follows -

$$
\operatorname{corr}(x, y)=\frac{\operatorname{cov}(x, y)}{S D(x) \times S D(y)}
$$

where $S D$ and $\operatorname{cov}(x, y)$ represent the standard deviation and covariance respectively. It lies in $[-1,1]$, with " -1 " and "1" indicate perfect negative and positive correlation respectively and, no relation when it approaches " 0 ".

For experiments, Lena image and its encrypted share, Ref. Fig. 3(a) and (b), are considered. The corr values in each of diagonal, horizontal and, vertical directions using randomly selected 2000 adjacent pixel pairs of $I$ are presented in Table 1. It is observed that correlation of $I$ is more than 0.92 , whereas, in Crypt-OR it falls close to 0 . It indicates that the proposed scheme efficiently breaks the pixels inter-correlation. Further, the corr values in Crypt-OR are compared with Chong et al. Fu et al. (2012), Teng et al. Teng \& Wang (2012) and, Tanwar et al. Tanwar et al. (2018b). 
Table 1: Comparison of correlation coefficient with existing schemes.

\begin{tabular}{|l|c|c|c|}
\hline \multicolumn{1}{|c|}{ Direction } & Horizontal & Vertical & Diagonal \\
\hline Secret Image & 0.9404 & 0.9299 & 0.9257 \\
\hline $\begin{array}{l}\text { Chong et al. } \\
\text { Fu et al. (2012) }\end{array}$ & 0.0088 & -0.0087 & 0.0060 \\
\hline $\begin{array}{l}\text { Teng et al. } \\
\text { Teng \& Wang (2012) }\end{array}$ & 0.0242 & -0.0194 & 0.0243 \\
\hline $\begin{array}{l}\text { Tanwar et al. } \\
\text { Tanwar et al. (2018b) }\end{array}$ & 0.0106 & 0.0112 & 0.0087 \\
\hline Crypto- $\boldsymbol{O R}$ & 0.0041 & -0.0084 & 0.0052 \\
\hline
\end{tabular}

\subsubsection{Visual analysis}

The high value of correlation coefficient (Eq. 16) indicate the compact relation bwteen the pixel intensity values and vice-versa for low correlation value. This compactness can be observed in first column of Fig. 6. The correlation of Lena gray-scale image of dimension $256 \times 256$ in each of vertical, horizontal, diagonal and anti-diagonal direction are shown in irst column of Fig. 6 (b), (c), (d) and (e) respectively. The corresponding correlation of encrypted image $I_{E n c}$ of Lena image in each directions are presented in second column of fig. 6. It indicates that the encrypted scheme efficiently secures the user image information from an adversary.

\subsection{Differential analysis}

Shamir's SSS adds a random number with each pixel intensity before generating the respective shares with varying prime numbers. With different random numbers, the encrypted form of secret image $I$ must change. The changes are observed using Unified Average Changing Intensity (UACI) and Number of Pixels Changing Rate (NPCR) Chen et al. (2004).

Consider two encrypted image $E_{1}^{I}$ and $E_{2}^{I}$, obtained before and after changing one pixel intensity of an $M \times N$-dimensional image $I$. Let $E_{1}^{I}(i, j)$ and 
(a)

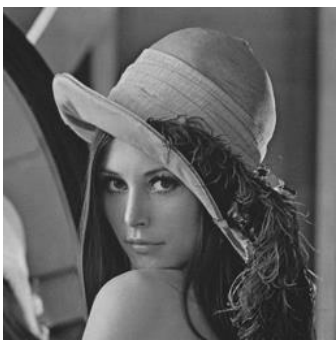

(b)
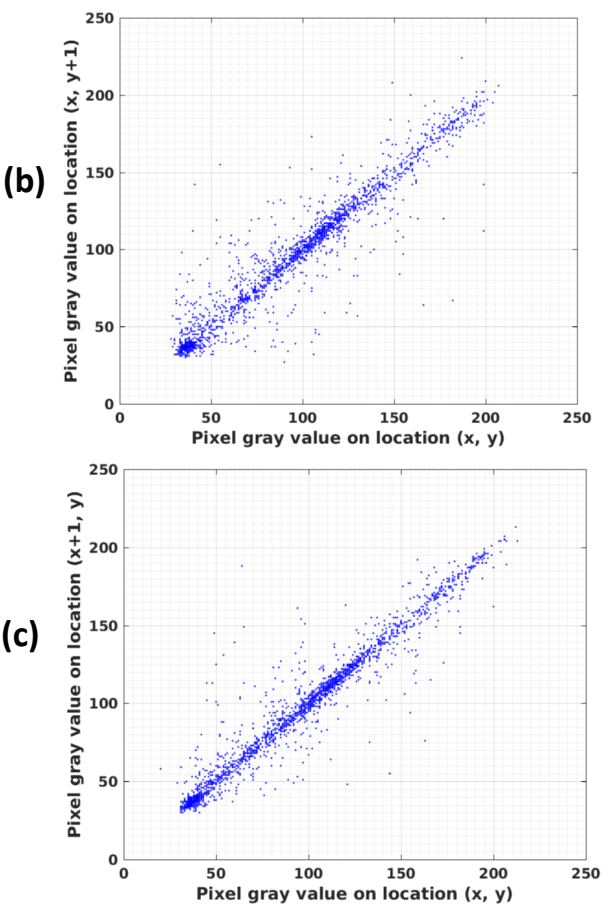
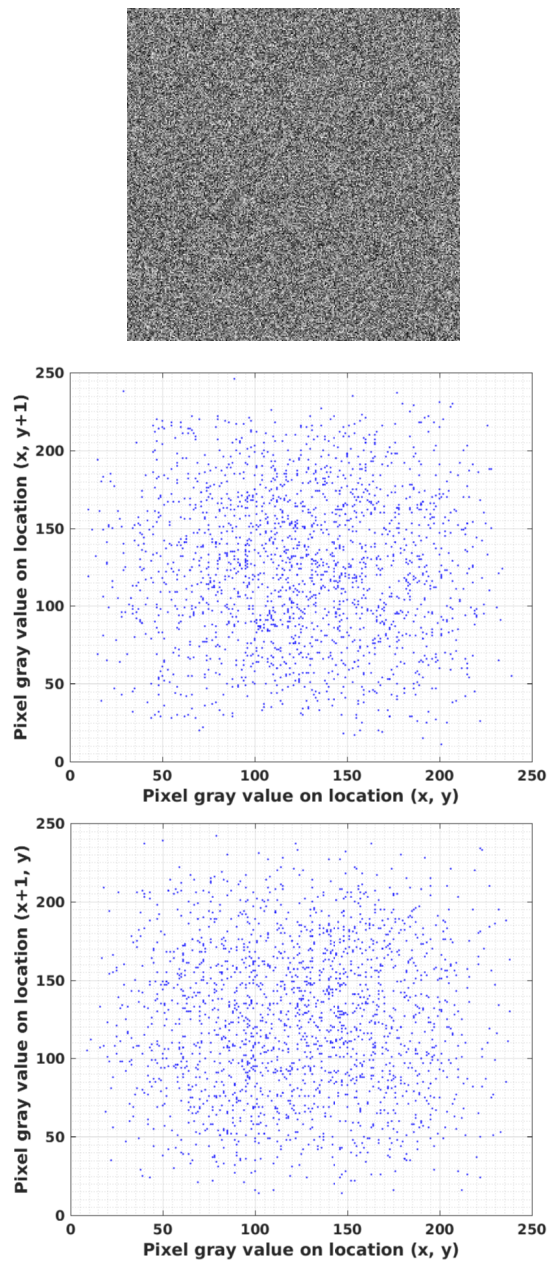

$E_{2}^{I}(i, j)$ denote the intensities at pixel $(i, j)^{t h}$-position in $E_{1}^{I}$ and $E_{2}^{I}$ respectively. Then, UACI and NPCR are calculated as follows -

$$
\begin{gathered}
\mathrm{UACI}=\frac{1}{M \times N}\left[\sum_{j=1}^{M} \sum_{i=1}^{N} \frac{\left|E_{1}^{I}(j, i)-E_{2}^{I}(j, i)\right|}{255}\right] \times 100 \% \\
\mathrm{NPCR}=\frac{1}{M \times N}\left[\sum_{j=1}^{M} \sum_{i=1}^{N} D(j, i)\right] \times 100 \%
\end{gathered}
$$

where, 

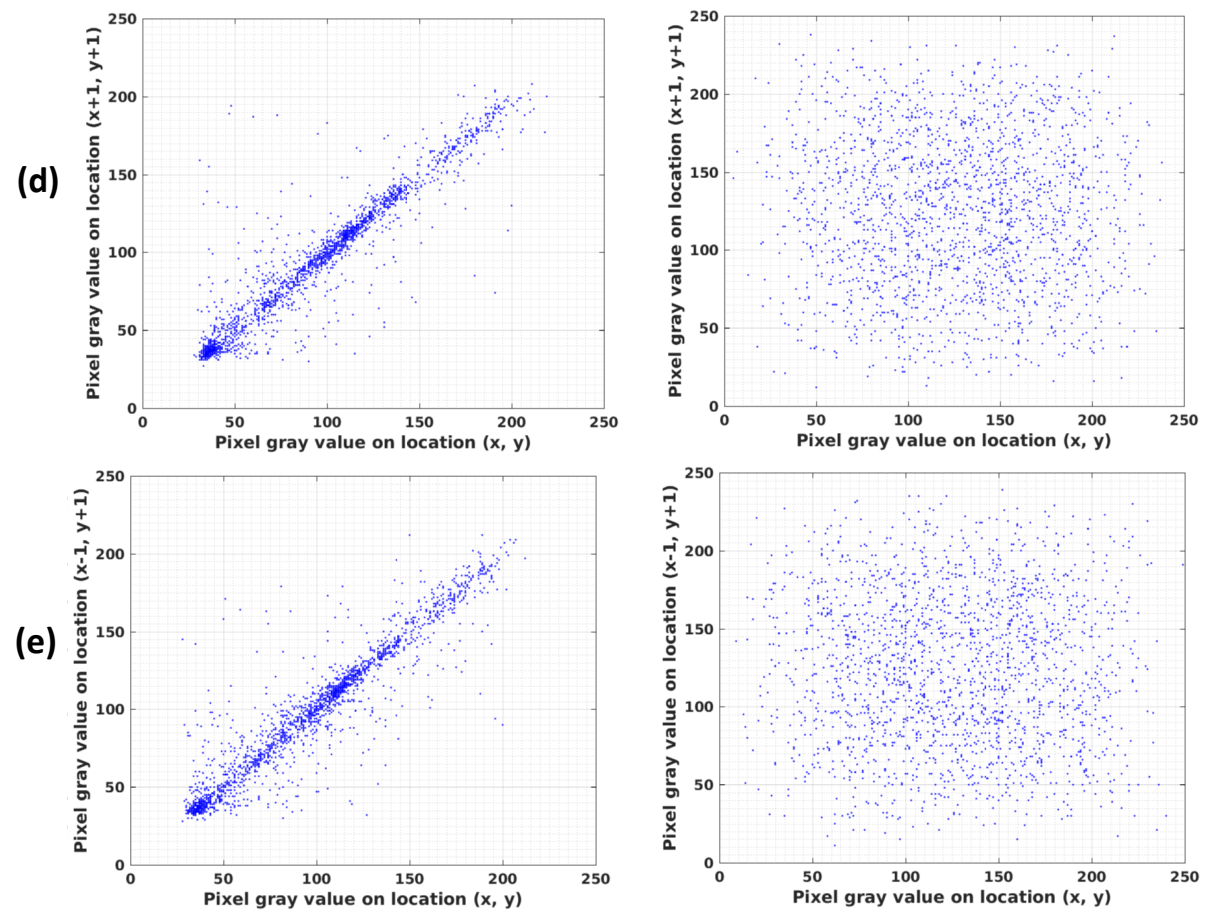

Figure 6: Comparison of correlation coefficient of (a) Lena image and its encrypted share in (b) horizontal, (c) vertical, (d) diagonal and, (e) anti-diagonal directions.

$$
D(j, i)= \begin{cases}1, & E_{1}^{I}(j, i) \neq E_{2}^{I}(j, i) \\ 0, & E_{1}^{I}(j, i)=E_{2}^{I}(j, i)\end{cases}
$$

The higher values of NPCR and UACI depict the high efficiency of the encryption algorithm to resist image information from differential attacks. For experiments, two shares of Lena image (Ref. Fig. 3 (a)) and the modified image obtained by interchanging two-pixels in each color channel at the same position are generated. Due to the addition of a random number, the average of 10 simultaneous NPCR and UACI values are reported as $\mathbf{9 9 . 9 5 2 0 \%}$ and $\mathbf{3 3 . 2 7 1 1 \%}$ respectively. The values determine that Crypt-OR can adequately 
resist differential attacks, and an eavesdropper cannot extract any information even after a modification in intensity values of the secret image.

\section{Experiments}

In this section, the perceptual and statistical results of Crypt-OR are prewith varying scenarios can be observed in the supplementary material.

Further, Crypto-OR is compared with the existing image inpainting and object-removal schemes presented by - Criminisi et al. Criminisi et al. (2004) 

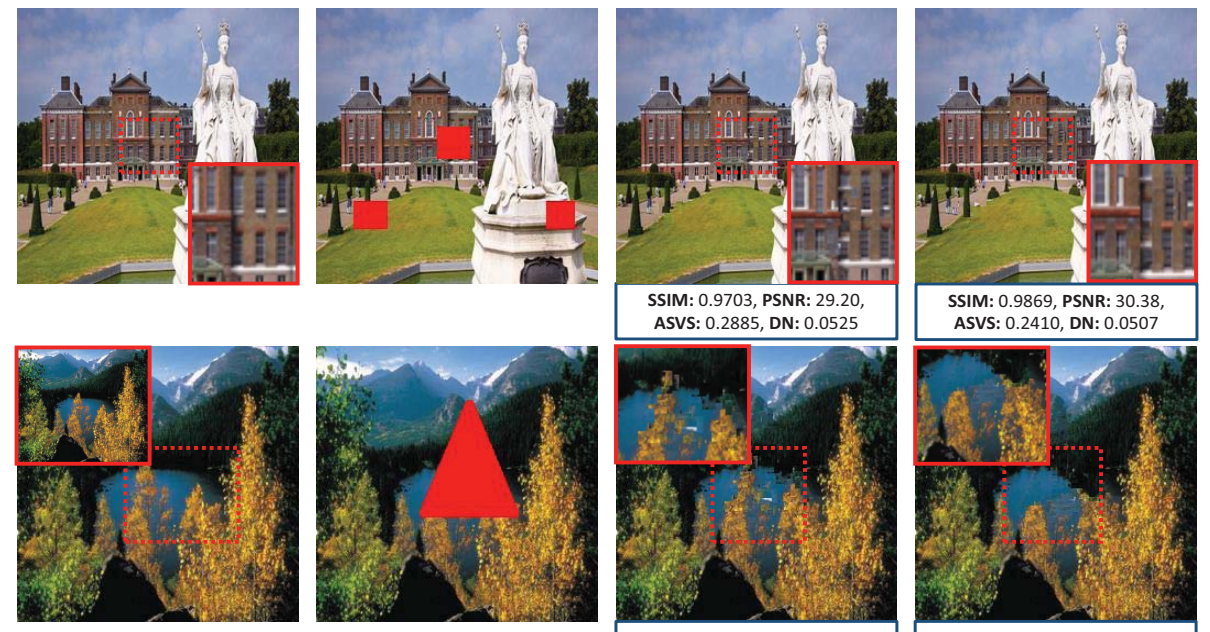

SSIM: 0.9556, PSNR: 33.11, ASVS: 0.2047, DN: 0.0412
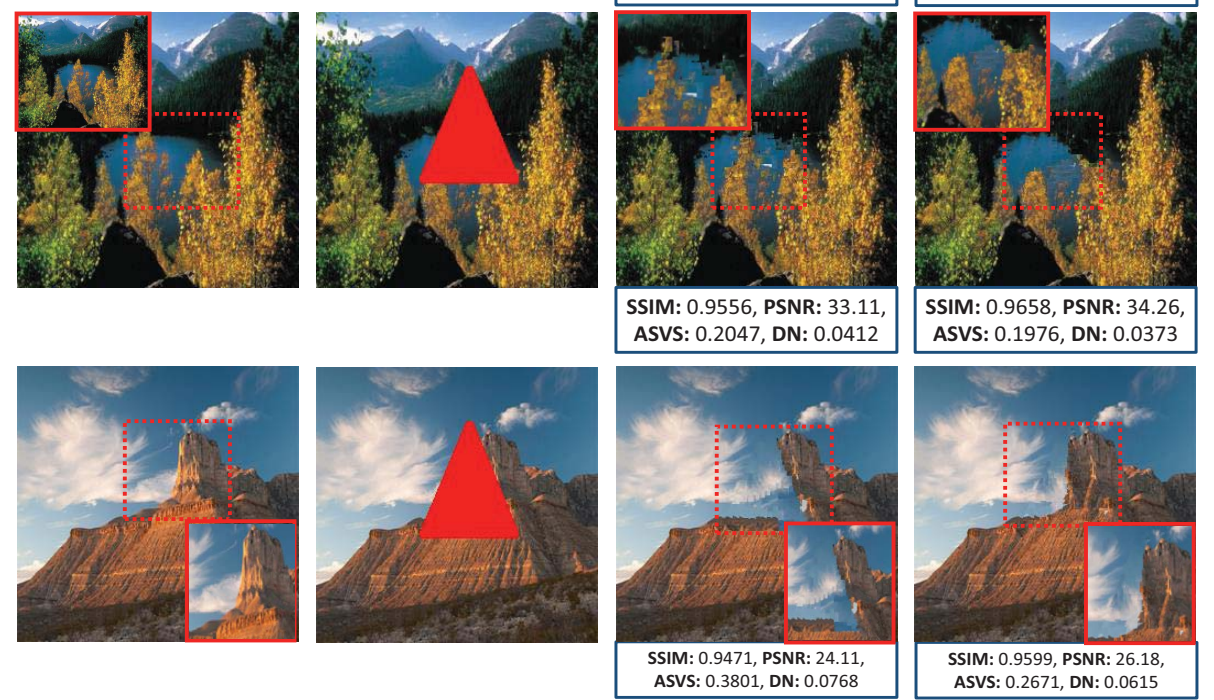

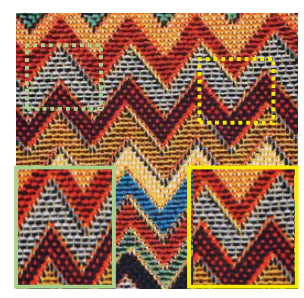

(a)

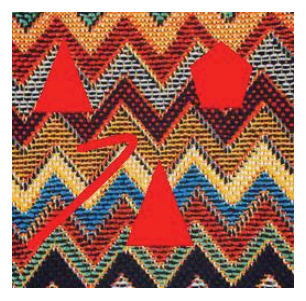

(b)

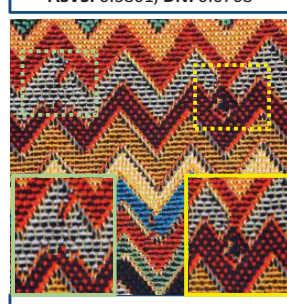

SSIM: 0.9673, PSNR: 36.80 ASVS: 0.1606, DN: 0.0229

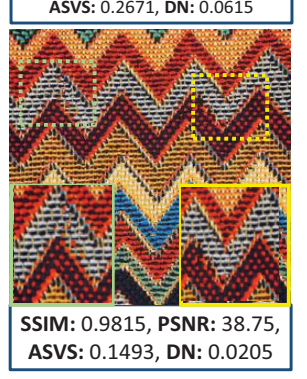

(c)

(d)

Figure 7: Comparison of the inpainted images in PD without and with regularizer in the data term. (a) Original image, (b) Superimposed object(s) to be removed are given in red color, (c) Baseline model, and (d) Baseline model with regularizer. The lined box(es) at the top/bottom of the image indicate the zoom-in portion of the dashed bounding-box(es). The impact of the regularizer can easily be observed in the lined-box(es) in (c) and (d), along with (a). 


\subsection{Quantitative analysis}

Here, the comparison of quantitative results of Crypt-OR with the existing inpainting schemes are presented. Due to the utilization of random numbers, while generating shares, each experiment is performed 10-times and their aver-

${ }^{4}$ https://github.com/knazeri/edge-connect ${ }^{5}$ https://github.com/JiahuiYu/generative_inpainting 
(a)

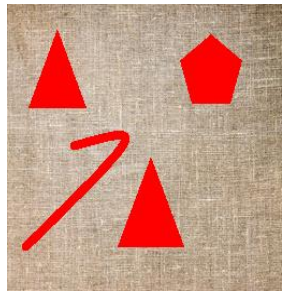

(b)
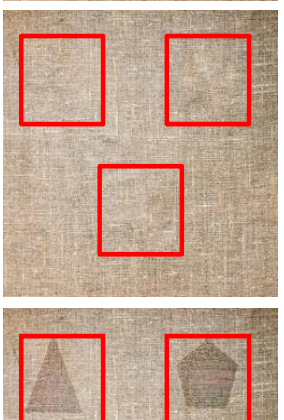

(c)
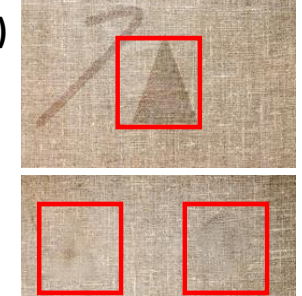

(d)
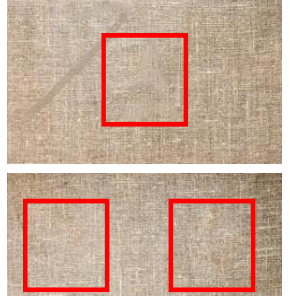

(e)

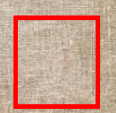

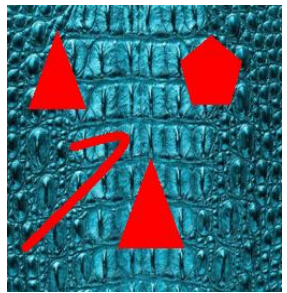
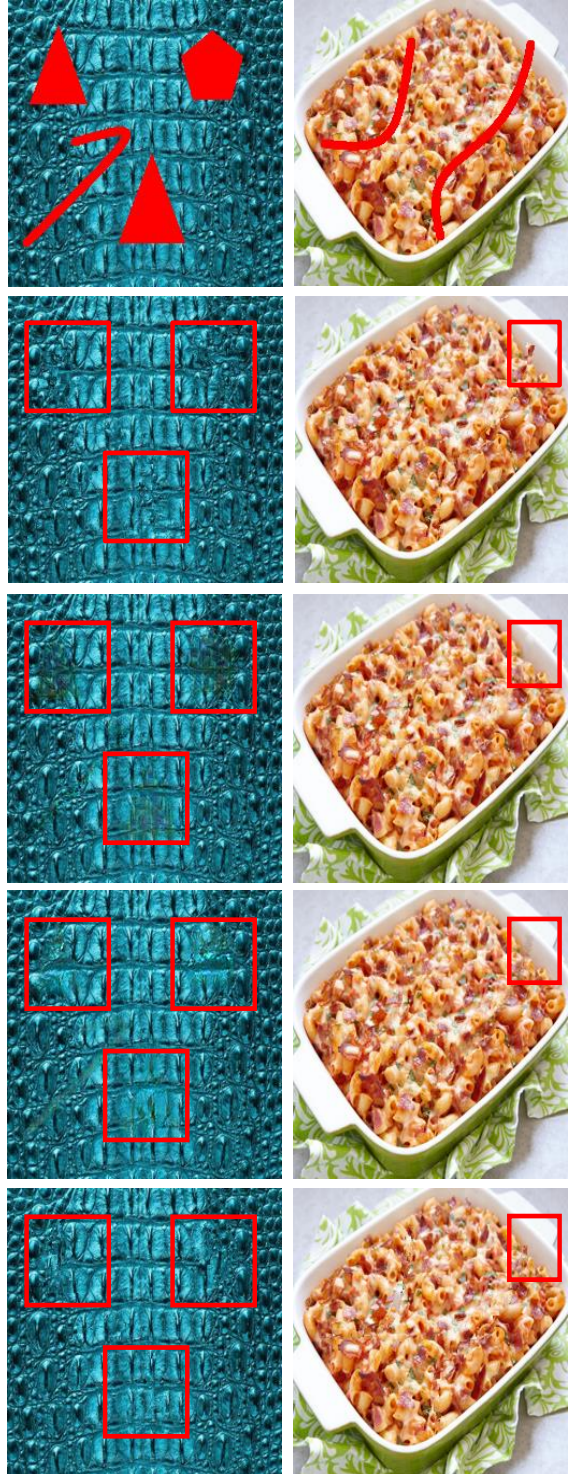
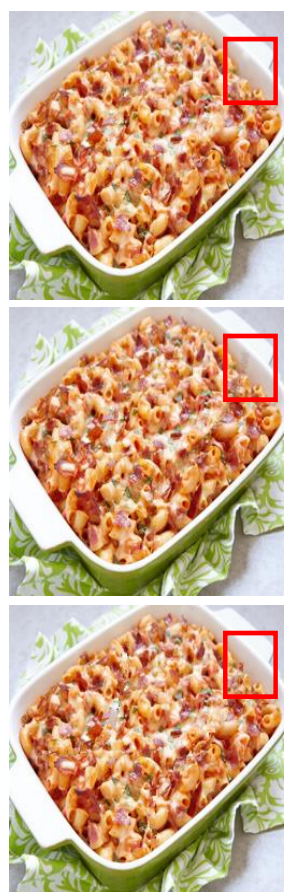
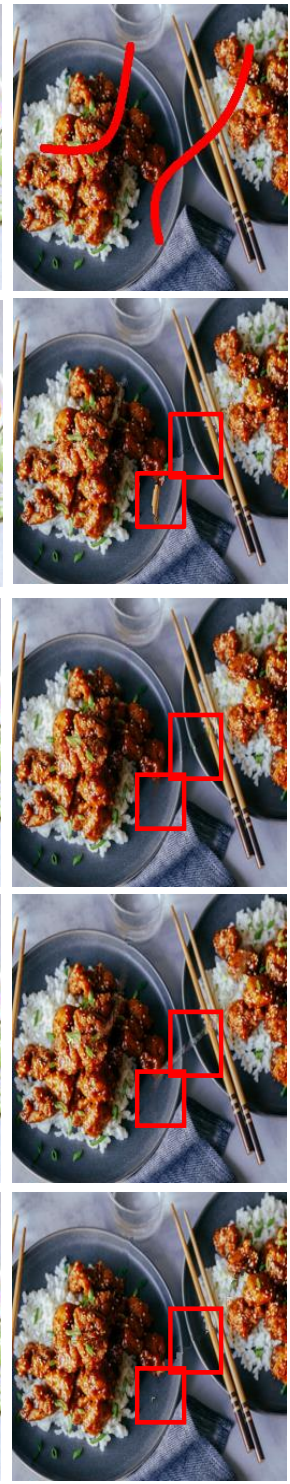

Figure 8: Comparison of Crypt-OR over texture and food images. (a) Original image - red indicates the undesired region(s), (b) Criminisi et al. Criminisi et al. (2004), (c) Nazeri et al. Nazeri et al. (2019), (d) Yu et al. Yu et al. (2018), and (e) Crypt-OR. The resulting difference in each scheme can be observed (top-to-down) by zoom-in the rectangle(s) region(s). 
(a)

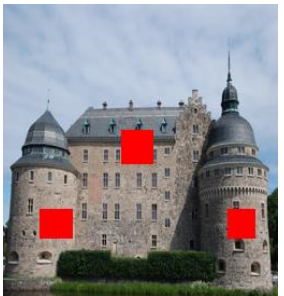

(b)

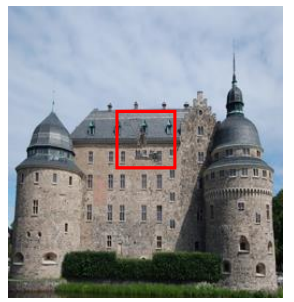

(c)

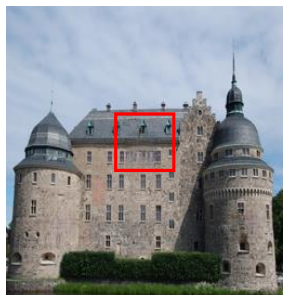

(d)
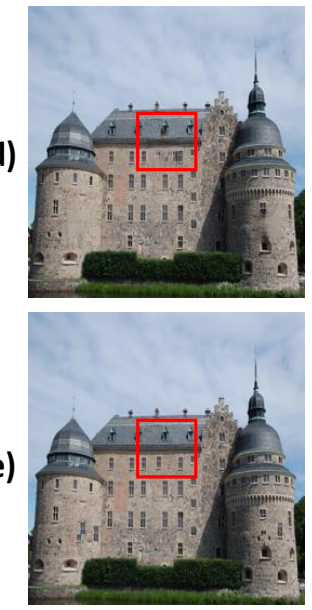
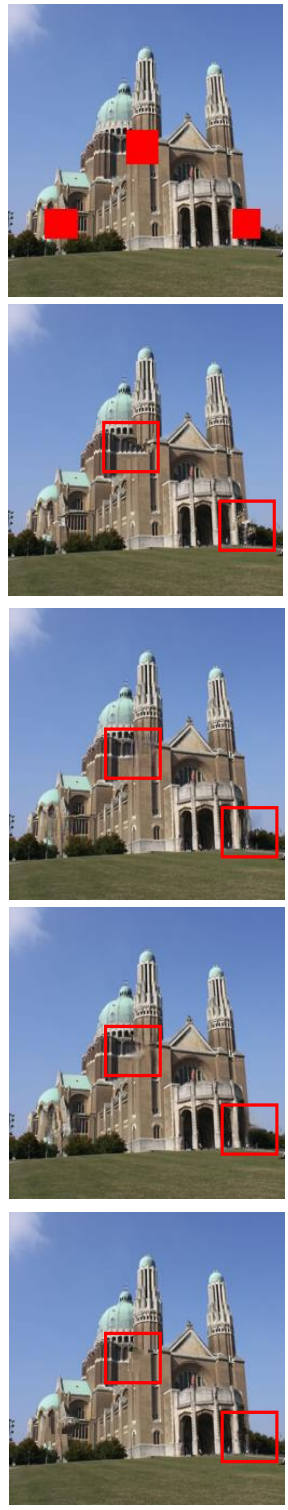
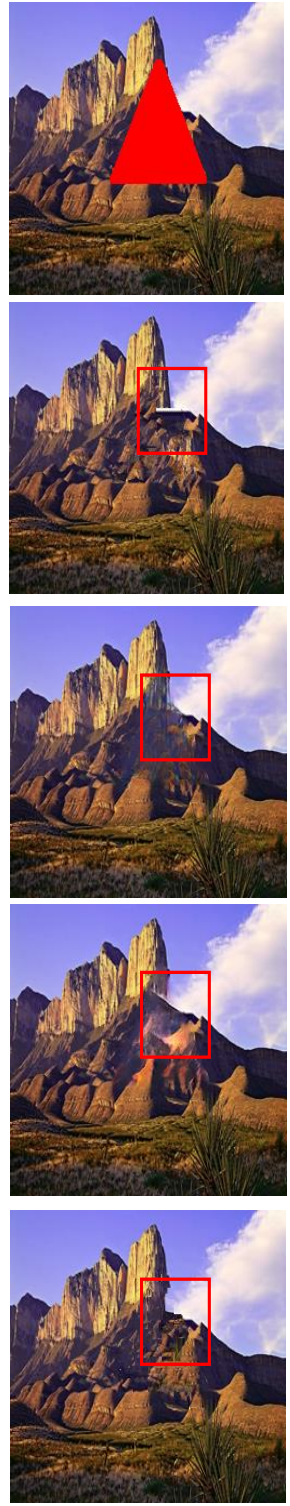
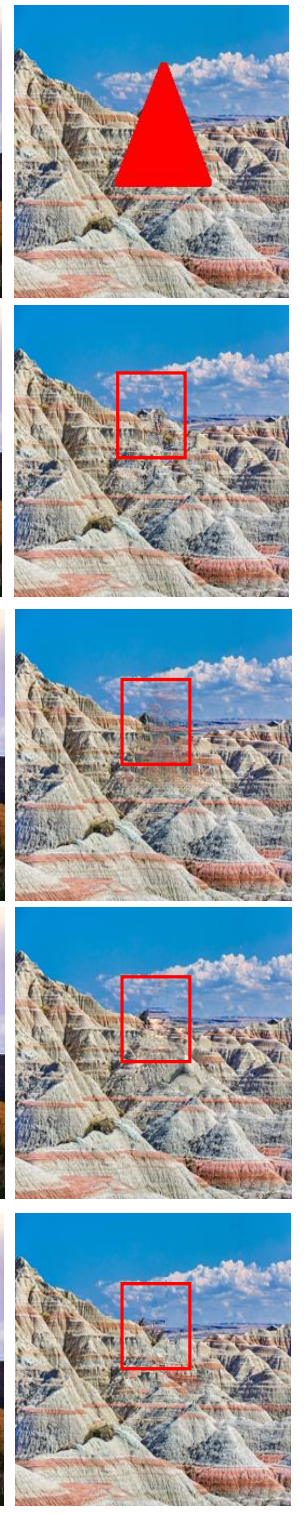

Figure 9: Comparison of Crypt-OR over monument and mountain images. (a) Original image - red indicates the undesired region(s), (b) Criminisi et al. Criminisi et al. (2004), (c) Nazeri et al. Nazeri et al. (2019), (d) Yu et al. Yu et al. (2018), and (e) Crypt-OR. The resulting difference in each scheme can be observed (top-to-down) by zoom-in the rectangle(s) region(s). 
6.2.1. Peak Signal-to-Noise Ratio (PSNR)

The PSNR between inpainted image $(I)$ and original image $(J)$ is evaluated using the following equation:

$$
P S N R=2 \times 10 \times \log _{10}(255)-10 \times \log _{10}(M S E)
$$

where MSE is mean squared error between $I$ and $J$.

The quality of the inpainted image increases with higher PSNR value, which is reported in Fig. 10, It can easily be observed that Crypt-OR outperforms Criminisi's model in all scenarios except mountains and close to Yu et al. and Nazir et al. models. The quality of an inpainted image obtained by Crypt-OR is close to the deep learning-based schemes in PD.

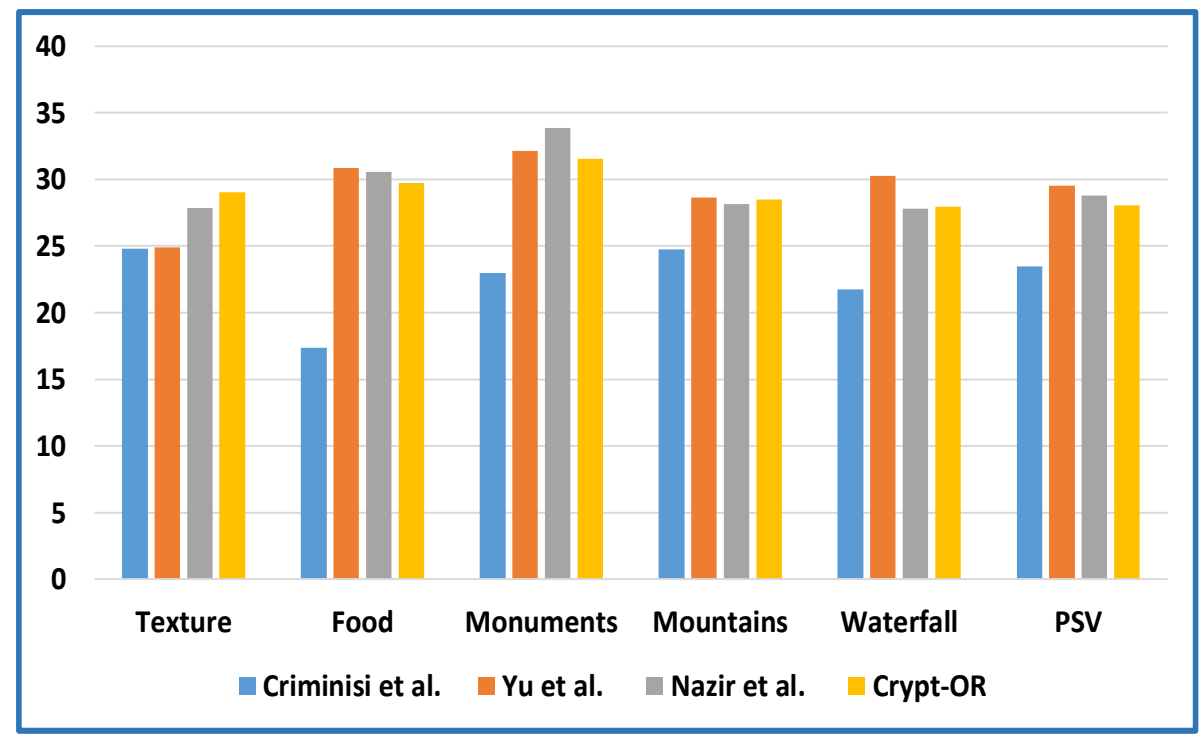

Figure 10: Comparison of PSNR values.

\subsubsection{Structural SIMilarity (SSIM) index}

The luminance, contrast and structural similarity Wu et al. (2013) between original image $(I)$ and inpainted image $(J)$ is compare by computing Mean SSIM 


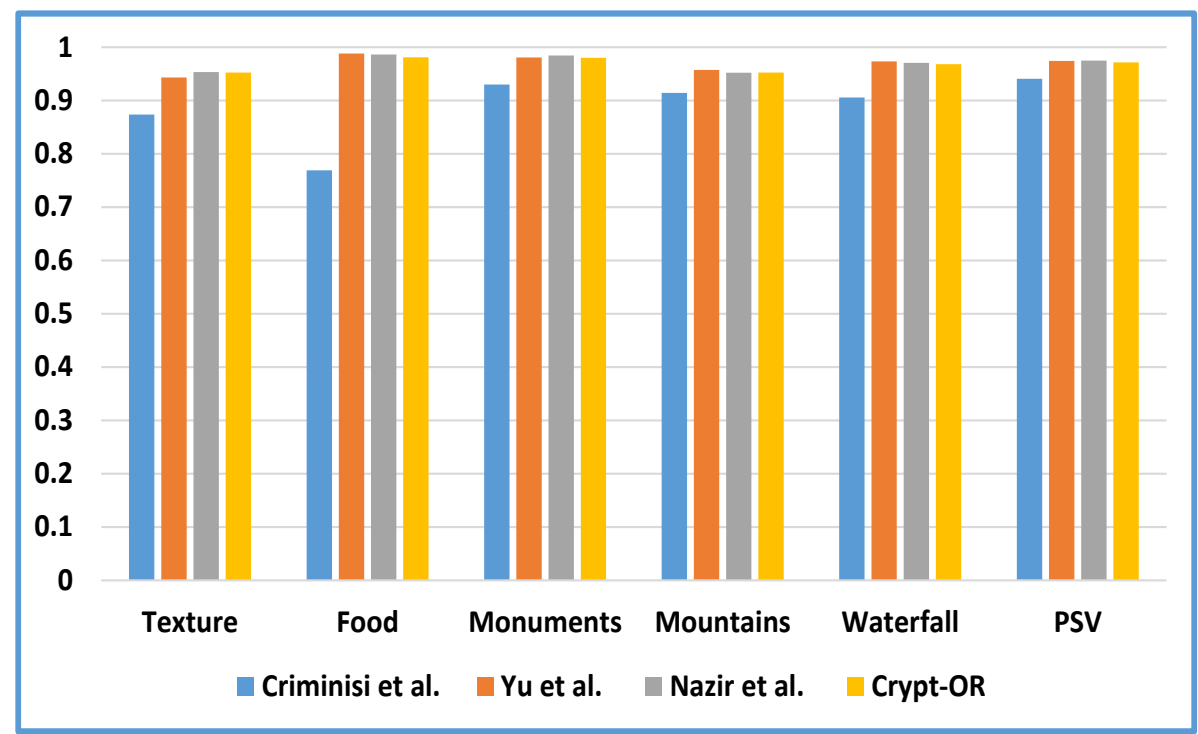

Figure 11: Comparison of SSIM values.

(MSSIM) index over $N_{w}$ number of local windows is computed as:

$$
M S S I M=\frac{1}{N_{w}} \sum_{k=1}^{N_{w}} \frac{\left(2 \mu_{I} \mu_{J}+C_{1}\right) \times\left(2 \sigma_{I J}+C_{2}\right)}{\left(\mu_{I}^{2}+\mu_{J}^{2}+C_{1}\right) \times\left(\sigma_{I}^{2}+\sigma_{J}^{2}+C_{2}\right)}
$$

where $\sigma_{k}$ and $\mu_{k}$ are standard deviation and average intensity value of $k \in\{I, J\} . \sigma_{I J}$ denotes correlation of $I$ and $J$ and, $C_{l}$ 's are stability constants. Further, $I_{k}$ and $J_{k}$ indicate the image patches for $k_{t h}$ window. It lies in the interval $[0,1]$, where the value near to 0 shows low structural similarity and that of 1 represents high structural similarity between $I$ and $J$.

The MSSIM values obtained between the original and inpainted images are depicted in Fig. 11. Crypt-OR outperforms Criminisi et al. (2004) in all scenarios and close to Nazeri et al. (2019) and Yu et al. (2018).

\subsubsection{Image inpainting quality measure}

The most reliable and accurate method to measure an image quality is the human-rating, the ultimate users. This measure is laborious, time consuming, and requires a large number of observers. Thus, a non-reference saliency-based 
inpainting metrics namely Average Squared Visual Salience (ASVS) and Degree of Noticeability (DN) proposed in Ardis \& Singhal (2009) are utilized.

These metrics compute the distortions in the inpainted image, which occurred due to the propagation of different colors, structures, and textures from the source region $\Phi$, to $\Omega$. ASVS computes the amount of noticeability of $\Omega$, in comparison with the whole image $I$, whereas DN measures the distortions that occurred in the neighborhood of $\Omega$, plus to distortions occurred inside $\Omega$. The mathematical definition is as follows -

$$
\begin{aligned}
& A S V S=\frac{1}{\|\Omega\|} \sum_{p \in \Omega}\left|S_{M}^{\prime}(p)\right|^{2} \times 100 \\
& D N=\frac{100}{\|\Omega\|+\|\Phi\|}\left(\sum_{p \in \Omega}\left|S_{M}^{\prime}(p)\right|^{2}+\sum_{p \in \Phi} E\left(S_{M}^{\prime}, S_{M}\right)\right) \\
& E\left(S_{M}^{\prime}, S_{M}\right)=\left|S_{M}^{\prime}(p)-S_{M}(p)\right|
\end{aligned}
$$

where, $S_{M}^{\prime}(p)$ depicts the salience map value of the inpainted pixel $p$ inside $\Omega$, in the inpainted image and $\|\Omega\|$ indicates the area of $\Omega$. The lower values of ASVS and DN represent the high perceptual quality of the inpainted image.

The average ASVS and DN values obtained by Crypt-OR are found to be comparable, except in mountain images, with the existing schemes Criminisi et al. (2004), Nazeri et al. (2019) and Yu et al. (2018) as shown in Fig. 12.

It can be observed that ASVS and DN values for Crypt-OR are less than the Criminisi et al. (2004) and equivalent to Nazeri et al. (2019); Yu et al. (2018), even lesser for images of texture, waterfall and PSV. Howbeit, it is high for mountain database. Similarly, DN values of Crypt-OR coincides with that of existing schemes Criminisi et al. (2004); Nazeri et al. (2019); Yu et al. (2018). In view of "No Free Lunch" theorem, Crypt-OR under-performs a bit for mountain database when compared with Nazeri et al. (2019); Yu et al. (2018), however, outperforms than Criminisi et al. (2004). The further analysis and comparison of Crypt-OR in view of structural similarity index and PSNR values are presented in supplementary material. 


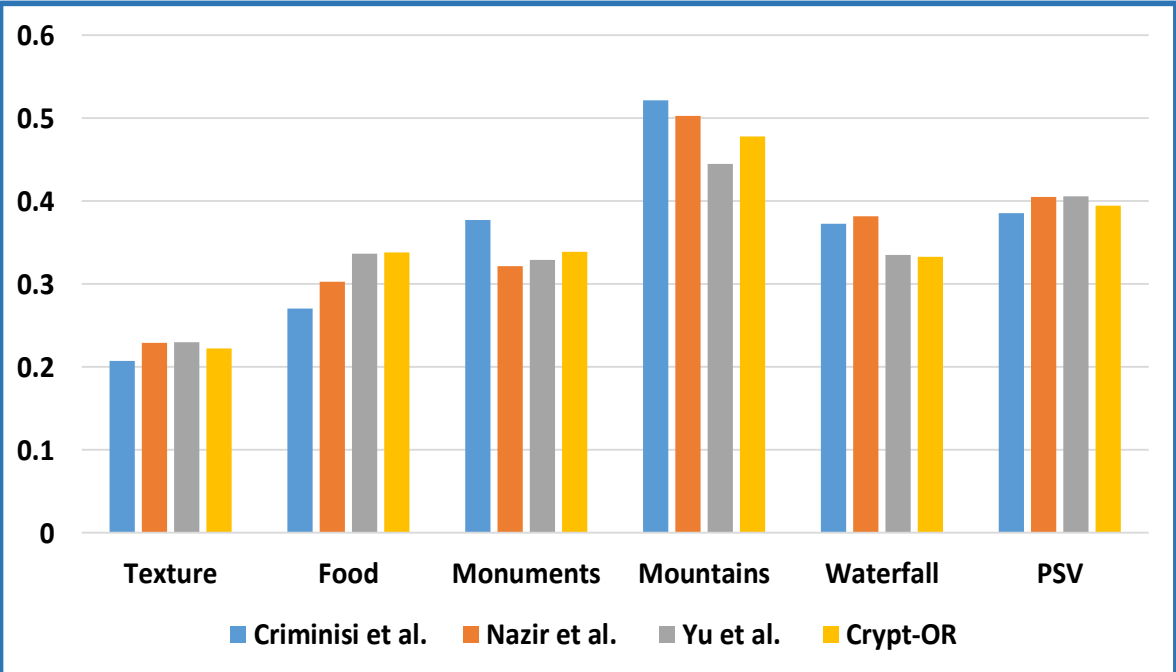

(a)

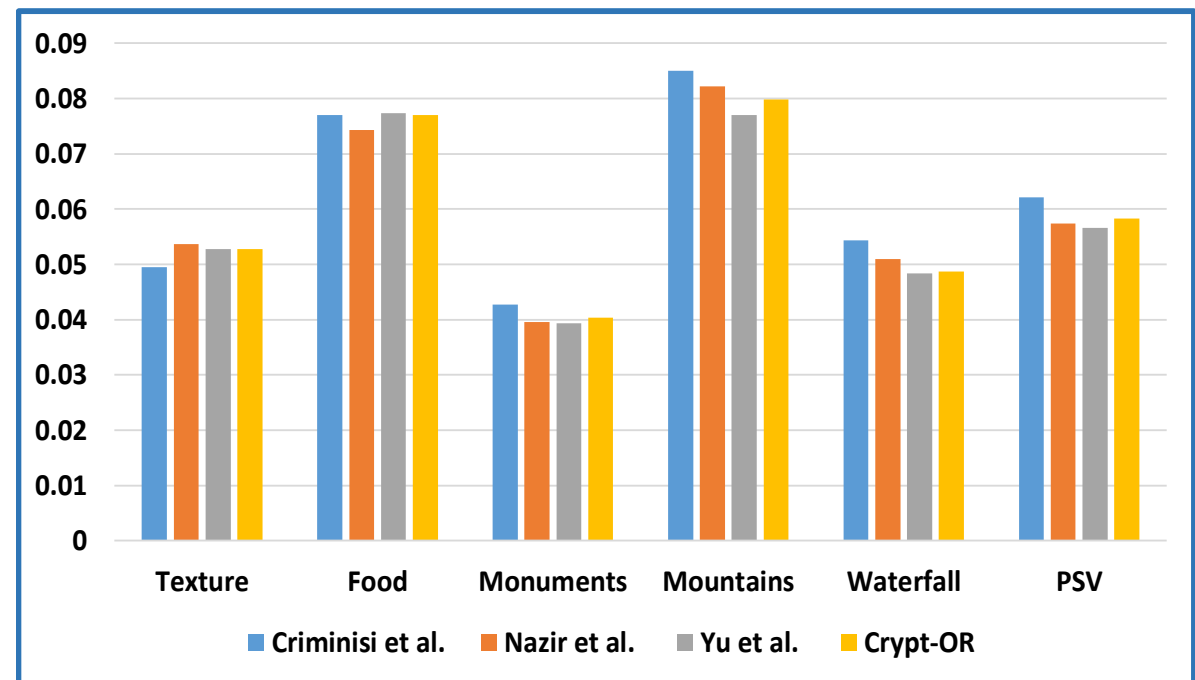

(b)

Figure 12: Comparison of (a) ASVS and, (b) DN values. 


\section{Discussion}

Here, the key outcomes of the proposed inpainting scheme are discussed by removing synthetically imposed shapes in grayscale images, as depicted in Fig. 13(a). It is observed that Criminisi's model Criminisi et al. (2004) can maintain the edges in an image, but also generates undesired information, except in the third row, Fig. 13(b). Nazeri et al. Nazeri et al. (2019) are unable to remove the specified region as the existing model was not trained for these shapes. Also, the neighboring background of the region has very little information and edges which the scheme can hallucinate, followed by completing the region. The inpainted images of Nazeri's model can be visualized in Fig. 13(c). Yu et al.

$545 \mathrm{Yu}$ et al. (2018) model is a generative approach that synthesizes the image structure and surrounding image features. It can be observed in Fig. 13(d) that the scheme fails to generate desirable results. Due to the non-availability of rich image features in the source region. The major drawbacks of these deep learning models are that they need to fine-tune/train over the new dataset with specific constraints as these are highly dependent upon the pixels inter-correlations. Also, they require high configured computing resources and storage space.

In contrast, Crypt-OR is a single-image based scheme and outperforms the existing schemes as depicted in Fig. 13 (e). It is observed that the proposed regularizer in data term and improved priority function generalize the behavior of structure and texture propagation in isophotes direction for the arbitrary region(s) to be inpaint. However, it is unable to propagate the edges of the triangle-shaped image (last-row), zoomed-in the upper corner of the triangle in Fig. 13(e), which is considered as future work.

\subsection{Performance analysis}

The computational cost required to remove the desired object(s) followed by fill-in the empty space using Crypt-OR consists of three processes - (i) encrypting the image into $N$ Shamir's shares, (ii) performing Crypt-OR over each share $S_{k}$ and, (iii) reconstruction of an inpainted image using $T$ inpainted shares. The processes (i) and (iii) are performed by the user and (ii) by CSP $C_{k}$. The total 

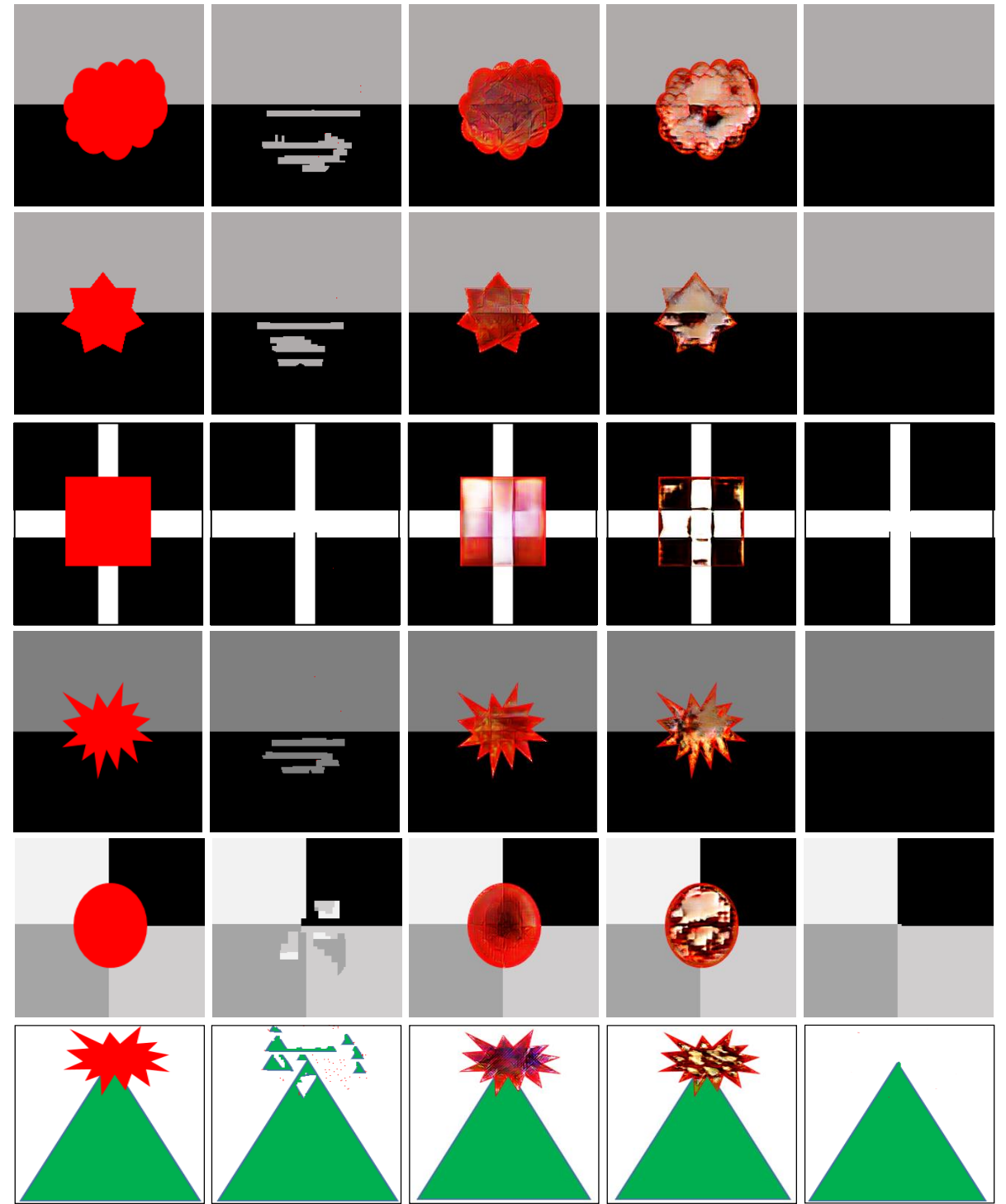

(a)

(b)

(c)

(d)

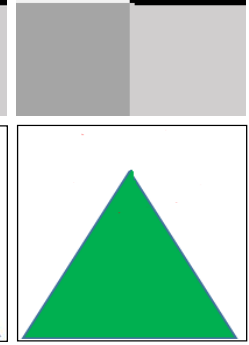

(e)

Figure 13: Comparison of Crypt-OR over various geometric shapes. (a) Original image - red indicates the region to be removed, (b) Criminisi et al. Criminisi et al. (2004), (c) Nazeri et al. Nazeri et al. (2019), (d) Yu et al. Yu et al. (2018), and (e) Crypt-OR. The effective results can be analyzed by zoom-in the inpainted region. 
Table 2: Performance analysis of object-removal in PD

\begin{tabular}{|c|c|c|c|c|}
\hline $\begin{array}{c}\text { Region } \\
\text { Percentage }\end{array}$ & \multicolumn{2}{|c|}{ Baseline model } & \multicolumn{2}{c|}{$\begin{array}{c}\text { Baseline with } \\
\text { regularizer }\end{array}$} \\
\hline & Min & Max & Min & Max \\
\hline $\mathbf{3} \mathbf{- 5} \%$ & 5.03 & 18.45 & 5.99 & 22.89 \\
\hline $\mathbf{6}-\mathbf{1 0} \%$ & 7.12 & 23.84 & 8.63 & 27.02 \\
\hline $\mathbf{1 1} \mathbf{- 1 5} \%$ & 10.78 & 29.57 & 12.92 & 34.35 \\
\hline
\end{tabular}

required-time for each process varies with the computational resources utilized by the user and CSP. For experimentation, we perform all three processes on our system, Ref. 6. The secret image of dimension $256 \times 256$ is shared into 4 Shamir's shares, which requires a time of 0.54 second. The decryption time using all 4 inpainted shares is 1.30 seconds. The inpainting time varies with the desired region-size and shape, as given in Table 2

\section{Conclusion and future work}

In this paper, a novel and efficient image inpainting model leveraging the merits of partial differential equations (PDEs) and exemplar-based schemes is proposed. A regularizer is introduced with the data-term for computing the 575 isophotes strength striking the boundary of the region. The scheme addresses the challenges of the small-sized object(s) in the depth of the image, varying textured-background, and objects(s) of specific geometric shapes such as triangle and rectangle. The scheme is further extended to perform in the ED over the cloud server as a SaaS. The secret image information is bifurcated into multiple shares of Shamir's SSS. The qualitative and quantitative results obtained

by Crypt-OR indicate the robust propagation of texture features and structure information in comparison with the existing schemes. The perceptual examples demonstrate that our scheme can be used for various applications such as removal of unwanted/undesired object(s), data hiding, and privacy surveillance. As future work, the removal of various facial features in a personal image in the ED over cloud infrastructures can be considered. 


\section{Acknowledgment}

This work is sponsored under grant: 21/12/2014(ii)EU-V, 2121440593 by University Grant Commission, INDIA.

590 Ardis, P. A., \& Singhal, A. (2009). Visual salience metrics for image inpainting. In Visual Communications and Image Processing (p. $72571 \mathrm{~W}$ ). International Society for Optics and Photonics volume 7257. doi $10.1117 / 12.808942$.

Arias, P., Facciolo, G., Caselles, V., \& Sapiro, G. (2011). A variational framework for exemplar-based image inpainting. International Journal of Computer Vision, 93, 319-347.

Bertalmio, M., Bertozzi, A. L., \& Sapiro, G. (2001). Navier-stokes, fluid dynamics, and image and video inpainting. In IEEE Computer Society Conference on Computer Vision and Pattern Recognition (pp. I-I). IEEE volume 1. doi:10.1109/CVPR.2001.990497.

Bertalmio, M., Sapiro, G., Caselles, V., \& Ballester, C. (2000). Image inpainting. In The Annual Conference on Computer Graphics and Interactive Techniques

口 (pp. 417-424). ACM Press/Addison-Wesley Publishing Co. doi 10.1145/ 344779.344972 .

Bost, R., Popa, R. A., Tu, S., \& Goldwasser, S. (2015). Machine learning classification over encrypted data. In NDSS.

Cao, C., Sun, K., \& Liu, W. (2018). A novel bit-level image encryption algorithm based on 2d-licm hyperchaotic map. Signal Processing, 143, 122-133.

Chen, G., Mao, Y., \& Chui, C. K. (2004). A symmetric image encryption scheme based on 3d chaotic cat maps. Chaos, Solitons \& Fractals, 21, 749-761.

Cheng, W.-H., Hsieh, C.-W., Lin, S.-K., Wang, C.-W., \& Wu, J.-L. (2005). Robust algorithm for exemplar-based image inpainting. In Proceedings of International Conference on Computer Graphics, Imaging and Visualization (pp. 64-69). 
Chu, K.-Y., Kuo, Y.-H., \& Hsu, W. H. (2013). Real-time privacy-preserving moving object detection in the cloud. In Proceedings of the ACM Inter1 national Conference on Multimedia (pp. 597-600). ACM. doi10.1145/ 2502081.2502157

Criminisi, A., Pérez, P., \& Toyama, K. (2004). Region filling and object removal by exemplar-based image inpainting. IEEE Transactions on Image Processing, 13, 1200-1212. doi 10.1109/TIP.2004.833105.

Drori, I., Cohen-Or, D., \& Yeshurun, H. (2003). Fragment-based image completion. In ACM Transactions on graphics (pp. 303-312). ACM volume 22.

Efros, A. A., \& Leung, T. K. (1999). Texture synthesis by non-parametric sampling. In International Conference on Computer Vision (pp. 1033-1038). IEEE volume 2.

Fu, C., Chen, J., Zou, H., Meng, W., Zhan, Y., \& Yu, Y. (2012). A chaos-based digital image encryption scheme with an improved diffusion strategy. Optics Express, 20, 2363-2378. doi:10.1364/0E.20.002363.

Gilad-Bachrach, R., Dowlin, N., Laine, K., Lauter, K., Naehrig, M., \& Wernsing, J. (2016). Cryptonets: Applying neural networks to encrypted data with high throughput and accuracy. In International Conference on Machine Learning (pp. 201-210).

Guillemot, C., \& Le Meur, O. (2013). Image inpainting: Overview and recent advances. Signal Processing Magazine, 31, 127-144.

${ }_{635}$ Guo, Y., Liu, Y., Oerlemans, A., Lao, S., Wu, S., \& Lew, M. S. (2016). Deep learning for visual understanding: A review. Neurocomputing, 187, 27-48.

Hsu, C.-Y., Lu, C.-S., \& Pei, S.-C. (2012). Image feature extraction in encrypted domain with privacy-preserving sift. IEEE Transactions on Image Processing, 21, 4593-4607. 
Jha, S., Kruger, L., \& McDaniel, P. (2005). Privacy preserving clustering. In European Symposium on Research in Computer Security (pp. 397-417). Springer. doi:10.1007/11555827_23.

Kaur, M., \& Kumar, V. (2018). A comprehensive review on image encryption techniques. Archives of Computational Methods in Engineering, (pp. 1-29).

Lathey, A., \& Atrey, P. K. (2015). Image enhancement in encrypted domain over cloud. ACM Transactions on Multimedia Computing, Communications, and Applications, 11, 38.

Lathey, A., Atrey, P. K., \& Joshi, N. (2013). Homomorphic low pass filtering on encrypted multimedia over cloud. In International Conference on Semantic 650 Computing (pp. 310-313). IEEE.

Liu, G., Reda, F. A., Shih, K. J., Wang, T.-C., Tao, A., \& Catanzaro, B. (2018). Image inpainting for irregular holes using partial convolutions. In Proceedings of the European Conference on Computer Vision (pp. 85-100).

Ma, X., Zhang, F., Chen, X., \& Shen, J. (2018). Privacy preserving multi-party 655 computation delegation for deep learning in cloud computing. Information Sciences, 459, 103-116.

Masnou, S., \& Morel, J.-M. (1998). Level lines based disocclusion. In International Conference on Image Processing (pp. 259-263). IEEE.

Mohanty, M., Ooi, W. T., \& Atrey, P. K. (2013). Scale me, crop me, knowme not: Supporting scaling and cropping in secret image sharing. In IEEE International Conference on Multimedia and Expo (pp. 1-6). IEEE. doi:10.1109/ICME. 2013.6607567.

Nazeri, K., Ng, E., Joseph, T., Qureshi, F., \& Ebrahimi, M. (2019). Edgeconnect: Structure guided image inpainting using edge prediction. In Proceedings of the IEEE International Conference on Computer Vision Workshops. 
Orlandi, C., Piva, A., \& Barni, M. (2007). Oblivious neural network computing via homomorphic encryption. EURASIP Journal on Information Security, $2007,037343$.

Padilla-López, J. R., Chaaraoui, A. A., \& Flórez-Revuelta, F. (2015). Visual privacy protection methods: A survey. Expert Systems with Applications, 42, $4177-4195$.

Pedrouzo-Ulloa, A., Troncoso-Pastoriza, J. R., \& Pérez-González, F. (2016). Image denoising in the encrypted domain. In International Workshop on Information Forensics and Security (pp. 1-6). IEEE. doi:10.1109/WIFS. 2016.7823916 .

Peter, P., Hoffmann, S., Nedwed, F., Hoeltgen, L., \& Weickert, J. (2016). Evaluating the true potential of diffusion-based inpainting in a compression context. Signal Processing: Image Communication, 46, 40-53.

Rahulamathavan, Y., Phan, R. C.-W., Chambers, J. A., \& Parish, D. J. (2013). Facial expression recognition in the encrypted domain based on local fisher discriminant analysis. IEEE Transactions on Affective Computing, 4, 83-92.

Rivest, R. L., Adleman, L., \& Dertouzos, M. L. (1978). On data banks and privacy homomorphisms. Foundations of Secure Computation, 4, 169-180.

SaghaianNejadEsfahani, S. M., Luo, Y., \& Cheung, S.-c. S. (2012). Privacy protected image denoising with secret shares. In IEEE International Conference on Image Processing (pp. 253-256). IEEE. doi:10.1109/ICIP. 2012.6466843.

Shamir, A. (1979). How to share a secret. Communications of the ACM, 22, 612-613. doi $10.1145 / 359168.359176$

Shokri, R., \& Shmatikov, V. (2015). Privacy-preserving deep learning. In ACM SIGSAC Conference on Computer and Communications Security (pp. 13101321). ACM. 
Tanwar, V. K., Rajput, A. S., Raman, B., \& Bhargava, R. (2018a). Privacy preserving image scaling using $2 \mathrm{~d}$ bicubic interpolation over the cloud. International Conference on Systems, Man, and Cybernetics, (pp. 2073-2078).

Tanwar, V. K., Rajput, A. S., Raman, B., \& Bhargava, R. (2018b). Privacy preserving image scaling using $2 \mathrm{~d}$ bicubic interpolation over the cloud. In International Conference on Systems, Man, and Cybernetics (pp. 2073-2078). IEEE.

Teng, L., \& Wang, X. (2012). A bit-level image encryption algorithm based on spatiotemporal chaotic system and self-adaptive. Optics Communications, 285, 4048-4054. doi:10.1016/j.optcom.2012.06.004.

Voulodimos, A., Doulamis, N., Doulamis, A., \& Protopapadakis, E. (2018). Deep learning for computer vision: A brief review. Computational intelligence and neuroscience, 2018.

Wang, M., Yan, B., \& Ngan, K. N. (2013). An efficient framework for image/video inpainting. Signal Processing: Image Communication, 28, 753762.

Wu, J., Lin, W., Shi, G., \& Liu, A. (2013). Reduced-reference image quality assessment with visual information fidelity. IEEE Transactions on Multimedia, 15, 1700-1705.

Xiang, S., Deng, H., Zhu, L., Wu, J., \& Yu, L. (2019). Exemplar-based depth inpainting with arbitrary-shape patches and cross-modal matching. Signal Processing: Image Communication, 71, 56-65.

Xie, P., Bilenko, M., Finley, T., Gilad-Bachrach, R., Lauter, K., \& Naehrig, M. ${ }_{715}$ (2014). Crypto-nets: Neural networks over encrypted data. arXiv preprint arXiv:1412.6181, .

Xing, K., Hu, C., Yu, J., Cheng, X., \& Zhang, F. (2017). Mutual privacy preserving $k$-means clustering in social participatory sensing. IEEE Transactions on Industrial Informatics, 13, 2066-2076. 
${ }_{720}$ Yan, W. Q., \& Kankanhalli, M. S. (2015). Face search in encrypted domain. In Pacific-rim Symposium on Image and Video Technology (pp. 775-790). Springer. doi:10.1007/978-3-319-29451-3_61.

Yan, X., Lu, Y., Liu, L., \& Wang, S. (2018). Partial secret image sharing for $(\mathrm{k}, \mathrm{n})$ threshold based on image inpainting. Journal of Visual Communi-

725 ㄴ cation and Image Representation, 50, 135-144. doi $10.1016 / j \cdot j v c i r .2017$. 11.012 .

Yonetani, R., Naresh Boddeti, V., Kitani, K. M., \& Sato, Y. (2017). Privacypreserving visual learning using doubly permuted homomorphic encryption. In International Conference on Computer Vision (pp. 2040-2050).

730 Yu, J., Lin, Z., Yang, J., Shen, X., Lu, X., \& Huang, T. S. (2018). Generative image inpainting with contextual attention. In IEEE Conference on Computer Vision and Pattern Recognition (pp. 5505-5514). 